\title{
Finitely connected vector spin systems with random matrix interactions
}

\author{
A C C Coolen ${ }^{1}$, N S Skantzos ${ }^{2}$, I Pérez Castillo ${ }^{3}$, C J Pérez Vicente ${ }^{4}$, \\ J P L Hatchett ${ }^{5}$, B Wemmenhove ${ }^{6}$ and T Nikoletopoulos ${ }^{1}$
}

${ }^{1}$ Department of Mathematics, King's College London, The Strand, London WC2R 2LS, UK

${ }^{2}$ Institute for Theoretical Physics, Celestijnenlaan 200D, Katholieke Universiteit Leuven, B-3001, Belgium

${ }^{3}$ Rudolf Peierls Center for Theoretical Physics, University of Oxford, 1 Keble Road, Oxford, OX1 3NP, UK

${ }^{4}$ Departament de Física Fonamental, Facultat de Física, Universitat de Barcelona, 08028 Barcelona, Spain

${ }^{5}$ Laboratory for Mathematical Neuroscience, RIKEN Brain Science Institute, Hirosawa 2-1, Wako-Shi, Saitama 351-0198, Japan

${ }^{6}$ Department of Medical Physics and Biophysics, Radboud University Nijmegen,

Geert Grooteplein 21, NL 6525 EZ Nijmegen, The Netherlands

E-mail: tcoolen@mth.kcl.ac.uk,Nikos.Skantzos@fys.kuleuven.ac.be, isaac@thphys.ox.ac.uk, conrad@ffn.ub.es, hatchett@brain.riken.jp,B.Wemmenhove@science.ru.nl and theodore@mth.kcl.ac.uk

Received 26 April 2005, in final form 5 July 2005

Published 14 September 2005

Online at stacks.iop.org/JPhysA/38/8289

\begin{abstract}
We use finite connectivity equilibrium replica theory to solve models of finitely connected unit-length vectorial spins, with random pair-interactions which are of the orthogonal matrix type. Finitely connected spin models, although still of a mean-field nature, can be regarded as a convenient level of description in between fully connected and finite-dimensional ones. Since the spins are continuous and the connectivity $c$ remains finite in the thermodynamic limit, the replica-symmetric order parameter is a functional. The general theory is developed for arbitrary values of the dimension $d$ of the spins, and arbitrary choices of the ensemble of random orthogonal matrices. We calculate phase diagrams and the values of moments of the order parameter explicitly for $d=2$ (finitely connected $X Y$ spins with random chiral interactions) and for $d=3$ (finitely connected classical Heisenberg spins with random chiral interactions). Numerical simulations are shown to support our predictions quite satisfactorily.
\end{abstract}

PACS numbers: $75.10 . \mathrm{Nr}, 05.20 .-\mathrm{y}, 64.60 . \mathrm{Cn}$ 


\section{Introduction}

Models of finitely connected disordered spin systems have been studied for some 20 years, following the initiating papers [1-5]. In particular due to the unexpectedly rich and varied range of multi-disciplinary applications of finite connectivity replica techniques which emerged subsequently in, for example, spin-glass modelling [6-9], error correcting codes [10-13], theoretical computer science [14-17], recurrent neural networks [18-20] and 'small-world' networks [21], this field is presently enjoying a renewed interest and popularity. Until very recently, analysis was limited to the equilibrium properties of such models, but now attention has also turned to the dynamics of finitely connected spin systems [22-25], using combinatorial and generating functional methods. In the domain of physical spin systems, research into finitely connected systems has usually been triggered by the desire to develop solvable spinglass models which are closer to real finite-dimensional systems than the celebrated fully connected spin-glass model of [26]. As far as we are aware, however (and in contrast to the situation with fully connected disordered spin systems), all finitely connected and disordered spin systems analysed theoretically so far involved scalar spin variables (either of the Ising type, the soft-spin type or the spherical type).

In the present paper, we solve equilibrium models of finitely connected spin systems of unit-length vectorial spins, and with random pair-interactions between them which are of a chiral nature, defined by random orthogonal matrices which promote random relative spatial orientations between pairs of spins. The motivation behind our study is twofold. Firstly, we aim to expand the domain of solvable and solved finitely connected spin models, by also including those where the spins have a truly vectorial character, and where their interactions are of a (random) matrix type. Vectorial spins are not only more realistic from a fundamental physical point of view in any real magnetic system, but are also of special relevance in the context of neural networks [27-29] (and more general models of coupled oscillators, see, e.g., the review [30]), and in describing granular superconductors and Josephson junctions [31-35]. In these latter junctions it is essential for the spin interactions to have a chiral character. In most such superconducting systems the local degrees of freedom are modelled as two-dimensional vectors, i.e., $X Y$ spins characterized by a single phase angle at each site. The random matrix interactions then induce random phase shifts between neighbouring sites; in two spatial dimensions one may subsequently find the so-called gauge glass state; see, e.g., [36-38] and references therein.

The inclusion of bond- and field-disorder in chiral spin systems has so far mostly been studied analytically in models where all spins are allowed to interact with each other (purely for mathematical convenience, see, e.g., [39] and references therein). The present study can be regarded as a step away from the unrealistic full connectivity towards finite-dimensional models in such vectorial systems. Our second motivation is of a technical nature. In contrast to models with discrete (e.g., Ising) spins, when choosing real-valued microscopic variables the replica-symmetric (RS) theory will involve an order parameter which is itself a functional, rather than a function. Solving the associated order parameter equations is therefore nontrivial, and leads to many numerical complications especially when the domain of values for the individual spins is not bounded (as is the case for, e.g., soft spins). In our present model we have vectorial spin states; although continuous and therefore leading to a theory involving an order parameter functional, each spin state represents a point on a sphere and has therefore a compact domain. This is found to be a considerable mathematical and numerical advantage, and allows us to push our analysis and therefore also our understanding further.

The structure of our paper is as follows. We first develop the general replica-symmetric theory for finitely connected vectorial spin systems with random chiral interactions described 
by orthogonal matrices, for arbitrary dimensions of the microscopic sphere $S_{d-1}$ which constrains the values of the individual spins. We then apply our theory first to the case $d=2$, where the spins reduce to $X Y$ ones, followed by application to $d=3$ where we have classical Heisenberg spins. In both cases, we calculate phase diagrams (with continuous transition lines calculated via functional moment expansions) as well as the values of macroscopic observables (using truncated population dynamics routines), for different choices for the ensemble of random orthogonal matrices. We complement our results with numerical simulations. The latter are found to agree well with our theoretical predictions, especially if the usual problems associated with the moment truncation of population dynamics algorithms and the well-known finite size effects in disordered spin systems are taken into account.

\section{Definitions}

We study finitely connected and bond-disordered systems comprising $N$ interacting normalized vectorial soft spins $\sigma_{i} \in S_{d-1}$, with $S_{d-1}$ denoting the unit sphere in $\mathbb{R}^{d}$. Thus for $d=2$ our spins become $X Y$ spins, with each spin representing a point on the unit circle, whereas for $d=3$ they become classical Heisenberg spins, with each representing a point on the unit sphere, etc. Our systems are taken to be in thermal equilibrium, characterized by Hamiltonians of the following form, with the short-hand $\{\sigma\}=\left(\sigma_{1}, \ldots, \sigma_{N}\right)$ :

$$
H(\{\boldsymbol{\sigma}\})=-J \sum_{i<j} c_{i j} \boldsymbol{\sigma}_{i} \cdot \mathbf{U}_{i j} \boldsymbol{\sigma}_{j}+\sum_{i} V\left(\boldsymbol{\sigma}_{i}\right)
$$

The independently distributed quenched random variables $c_{i j} \in\{0,1\}$ define the (random) connectivity of the system, i.e., they dictate which pairs of spins are allowed to interact. The real $d \times d$ matrices are assumed orthogonal and with determinant 1 , i.e., they represent rotations in $\mathbb{R}^{d}$, and are drawn randomly and independently from some random matrix ensemble characterized by a distribution $P(\mathbf{U})$. We will assume as yet only that $P(\mathbf{U})=P\left(\mathbf{U}^{\dagger}\right)$. The single-site potentials $V(\sigma)$ serve to break symmetries, and will also enable us to take an Ising limit later as a benchmark test (e.g., for $V(\boldsymbol{\sigma})=\gamma(\hat{\boldsymbol{e}} \cdot \boldsymbol{\sigma})^{2}$, with $\hat{\boldsymbol{e}}$ denoting a fixed unit-length vector in $\mathbb{R}^{d}$, and where $\gamma \rightarrow \infty$ ). In this paper, we will be concerned with the so-called finite connectivity regime, where

$$
\operatorname{Prob}\left(c_{i j}\right)=\frac{c}{N} \delta_{c_{i j}, 1}+\left(1-\frac{c}{N}\right) \delta_{c_{i j}, 0} \quad \text { for all } \quad i<j
$$

with $c=\mathcal{O}\left(N^{0}\right)$. Each spin on an Erdös-Rényi graph generated via (2) interacts on average only with a finite number $c$ of other spins, even in the thermodynamic limit, similar to finitedimensional systems. In this sense, in spite of the absence of geometrical considerations (so that they are still of a mean-field nature), finitely connected spin models can be regarded as closer to physical reality than fully connected ones.

In the remainder of this paper, averages over the random connectivity $\left\{c_{i j}\right\}$ and over the random orthogonal matrices $\left\{\mathbf{U}_{i j}\right\}$ will be denoted by $\cdots$. We will use the standard replica identity $\overline{\log Z}=\lim _{n \rightarrow 0} n^{-1} \log \overline{Z^{n}}$, with $\overline{Z^{n}}$ initially evaluated for integer $n$, to calculate for our system the asymptotic disorder-averaged free energy per spin $\bar{f}=$ $-\lim _{N \rightarrow \infty}(\beta N)^{-1} \overline{\log Z}$. Indices will be used according to the standard conventions, with Greek ones labelling replicas $(\alpha=1, \ldots, n)$ and Roman ones labelling spins $(i=1, \ldots, N)$. 


\section{Replica calculation of the disorder-averaged free energy per spin}

\subsection{Derivation of replica saddle-point equations}

In evaluating the disorder-averaged free energy per spin with the replica method,

$$
\bar{f}=-\lim _{N \rightarrow \infty} \frac{1}{\beta N} \overline{\left[\log \int_{S_{d-1}}\left[\prod_{i} \mathrm{~d} \sigma_{i}\right] \mathrm{e}^{-\beta H(\{\sigma\})}\right]}
$$

one finds that site factorization can be achieved upon isolating the usual site-averaged replica order parameter for finitely connected systems. After redefining $\{\sigma\}=\left(\sigma^{1}, \ldots, \sigma_{n}\right)$, where now $\sigma_{i}^{\alpha}, \sigma_{\alpha} \in S_{d-1}$, this order parameter takes the form $P(\{\boldsymbol{\sigma}\})=\lim _{N \rightarrow \infty} N^{-1}$ $\sum_{i} \overline{\prod_{\alpha} \delta\left[\sigma^{\alpha}-\sigma_{i}^{\alpha}\right]}$. The insertion of appropriate functional $\delta$-distributions into (3) gives

$$
\begin{aligned}
\bar{f}= & -\lim _{N \rightarrow \infty} \lim _{n \rightarrow 0} \frac{1}{\beta N n} \log \int \cdots \int_{S_{d-1}}\left[\prod_{i \alpha} \mathrm{d} \boldsymbol{\sigma}_{i}^{\alpha} \mathrm{e}^{-\beta V\left(\boldsymbol{\sigma}_{i}^{\alpha}\right)}\right] \prod_{i<j} \overline{\exp \left(\beta J c_{i j} \sum_{\alpha} \boldsymbol{\sigma}_{i}^{\alpha} \cdot \mathbf{U}_{i j} \boldsymbol{\sigma}_{j}^{\alpha}\right)} \\
= & -\lim _{N \rightarrow \infty} \lim _{n \rightarrow 0} \frac{1}{\beta N n} \log \int \cdots \int_{S_{d-1}}\left[\prod_{i \alpha} \mathrm{d} \boldsymbol{\sigma}_{i}^{\alpha} \mathrm{e}^{-\beta V\left(\boldsymbol{\sigma}_{i}^{\alpha}\right)}\right] \\
& \times \exp \left\{\frac{c}{2 N} \sum_{i j}\left[\int \mathrm{d} \mathbf{U} P(\mathbf{U}) \exp \left(\beta J \sum_{\alpha} \boldsymbol{\sigma}_{i}^{\alpha} \cdot \mathbf{U} \boldsymbol{\sigma}_{j}^{\alpha}\right)-1\right]+\mathcal{O}\left(N^{0}\right)\right\} \\
= & -\lim _{N \rightarrow \infty} \lim _{n \rightarrow 0} \frac{1}{\beta N n} \log \int\left\{\prod_{\{\boldsymbol{\sigma}\}} \frac{\mathrm{d} P(\{\boldsymbol{\sigma}\}) \mathrm{d} \hat{P}(\{\boldsymbol{\sigma}\}) \exp (\mathrm{i} N P(\{\boldsymbol{\sigma}\}) \hat{P}(\{\boldsymbol{\sigma}\}))}{2 \pi / N}\right\} \\
& \times \exp \left\{\frac{1}{2} c N \int\left\{\mathrm{d} \boldsymbol{\sigma} \mathrm{d} \boldsymbol{\sigma}^{\prime}\right\} P(\{\boldsymbol{\sigma}\}) P\left(\left\{\boldsymbol{\sigma}^{\prime}\right\}\right)\left[\int \mathrm{d} \mathbf{U} P(\mathbf{U}) \exp \left(\beta J \sum_{\alpha} \boldsymbol{\sigma}_{\alpha} \cdot \mathbf{U} \boldsymbol{\sigma}_{\alpha}^{\prime}\right)-1\right]\right\} \\
& \times \exp \left\{N \log \int\{\mathrm{d} \boldsymbol{\sigma}\} \exp \left(-\mathrm{i} \sum_{\left\{\boldsymbol{\sigma}^{\prime}\right\}} \hat{P}\left(\left\{\boldsymbol{\sigma}^{\prime}\right\}\right) \prod \prod_{\alpha} \delta\left[\boldsymbol{\sigma}_{\alpha}^{\prime}-\boldsymbol{\sigma}_{\alpha}\right]-\beta \sum_{\alpha} V\left(\boldsymbol{\sigma}_{\alpha}\right)\right)\right\}
\end{aligned}
$$

Next we carry out a rescaling transformation of the conjugate integration variables: $\hat{P}(\{\sigma\}) \rightarrow$ $\{\mathrm{d} \sigma\} \hat{P}(\{\sigma\})$. The objective is to convert the sum $\sum_{\{\sigma\}} P(\{\sigma\}) \hat{P}(\{\sigma\})$ in the exponent into $\sum_{\{\sigma\}}\{\mathrm{d} \sigma\} P(\{\sigma\}) \hat{P}(\{\sigma\})$; this latter expression, in contrast to the original one, will become a well-defined integral in the continuum limit $\{\mathrm{d} \boldsymbol{\sigma}\} \rightarrow\{\mathbf{0}\}$. Upon writing the resulting path integration measure as $\prod_{\{\sigma\}}[\mathrm{d} P(\{\sigma\}) \mathrm{d} \hat{P}(\{\sigma\}) / 2 \pi]=\{\mathrm{d} P \mathrm{~d} \hat{P}\}$, and upon neglecting irrelevant constants, our expression for the disorder-averaged free energy per spin is then seen to take a saddle-point form:

$$
\begin{aligned}
\bar{f}= & -\lim _{N \rightarrow \infty} \lim _{n \rightarrow 0} \frac{1}{\beta N n} \log \int\{\mathrm{d} P \mathrm{~d} \hat{P}\} \exp \left(\mathrm{i} N \int\{\mathrm{d} \boldsymbol{\sigma}\} P(\{\boldsymbol{\sigma}\}) \hat{P}(\{\boldsymbol{\sigma}\})\right) \\
& \times \exp \left\{\frac{1}{2} c N \int\left\{\mathrm{d} \boldsymbol{\sigma} \mathrm{d} \boldsymbol{\sigma}^{\prime}\right\} P(\{\boldsymbol{\sigma}\}) P\left(\left\{\boldsymbol{\sigma}^{\prime}\right\}\right)\left[\int \mathrm{d} \mathbf{U} P(\mathbf{U}) \exp \left(\beta J \sum_{\alpha} \boldsymbol{\sigma}_{\alpha} \cdot \mathbf{U} \boldsymbol{\sigma}_{\alpha}^{\prime}\right)-1\right]\right\} \\
& \times \exp \left\{N \log \int\{\mathrm{d} \boldsymbol{\sigma}\} \exp \left(-\beta \sum_{\alpha} V\left(\boldsymbol{\sigma}_{\alpha}\right)-\mathrm{i} \hat{P}(\{\boldsymbol{\sigma}\})\right)\right\}
\end{aligned}
$$




$$
\begin{aligned}
= & -\lim _{n \rightarrow 0} \frac{1}{\beta n} \operatorname{extr}_{\{P, \hat{P}\}}\left\{\mathrm{i} \int\{\mathrm{d} \boldsymbol{\sigma}\} P(\{\boldsymbol{\sigma}\}) \hat{P}(\{\boldsymbol{\sigma}\})+\log \int\{\mathrm{d} \boldsymbol{\sigma}\} \exp \left(-\beta \sum_{\alpha} V\left(\boldsymbol{\sigma}_{\alpha}\right)-\mathrm{i} \hat{P}(\{\boldsymbol{\sigma}\})\right)\right. \\
& \left.+\frac{1}{2} c \int\left\{\mathrm{d} \boldsymbol{\sigma} \mathrm{d} \boldsymbol{\sigma}^{\prime}\right\} P(\{\boldsymbol{\sigma}\}) P\left(\left\{\boldsymbol{\sigma}^{\prime}\right\}\right)\left[\int \mathrm{d} \mathbf{U} P(\mathbf{U}) \exp \left(\beta J \sum_{\alpha} \boldsymbol{\sigma}_{\alpha} \cdot \mathbf{U} \boldsymbol{\sigma}_{\alpha}^{\prime}\right)-1\right]\right\} .
\end{aligned}
$$

The functional variation of (4) with respect to the two order parameter functions $\hat{P}(\{\sigma\})$ and $P(\{\sigma\})$ gives us the following two saddle-point equations, respectively:

$$
\begin{aligned}
& P(\{\boldsymbol{\sigma}\})=\frac{\exp \left(-\beta \sum_{\alpha} V\left(\boldsymbol{\sigma}_{\alpha}\right)-\mathrm{i} \hat{P}(\{\boldsymbol{\sigma}\})\right)}{\int\left\{\mathrm{d} \boldsymbol{\sigma}^{\prime}\right\} \exp \left(-\beta \sum_{\alpha} V\left(\boldsymbol{\sigma}_{\alpha}^{\prime}\right)-\mathrm{i} \hat{P}\left(\left\{\boldsymbol{\sigma}^{\prime}\right\}\right)\right)} \\
& \hat{P}(\{\boldsymbol{\sigma}\})=\mathrm{i} c \int\left\{\mathrm{d} \boldsymbol{\sigma}^{\prime}\right\} P\left(\left\{\boldsymbol{\sigma}^{\prime}\right\}\right)\left[\int \mathrm{d} \mathbf{U} P(\mathbf{U}) \exp \left(\beta J \sum_{\alpha} \boldsymbol{\sigma}_{\alpha} \cdot \mathbf{U} \boldsymbol{\sigma}_{\alpha}^{\prime}\right)-1\right] .
\end{aligned}
$$

Elimination of the conjugate order parameter function $\hat{P}(\{\sigma\})$ leads to a saddle-point equation for $P(\{\sigma\})$ only, and an associated expression for the free energy $\bar{f}$ :

$$
\begin{gathered}
P(\{\boldsymbol{\sigma}\})=\frac{\exp \left(c \int\left\{\mathrm{d} \boldsymbol{\sigma}^{\prime}\right\} P\left(\left\{\boldsymbol{\sigma}^{\prime}\right\}\right)\left[\int \mathrm{d} \mathbf{U} P(\mathbf{U}) \mathrm{e}^{\beta J \sum_{\alpha} \boldsymbol{\sigma}_{\alpha} \cdot \mathbf{U} \boldsymbol{\sigma}_{\alpha}^{\prime}}-1\right]-\beta \sum_{\alpha} V\left(\boldsymbol{\sigma}_{\alpha}\right)\right)}{\int\left\{\mathrm{d} \boldsymbol{\sigma}^{\prime}\right\} \exp \left(c \int\left\{\mathrm{d} \boldsymbol{\sigma}^{\prime \prime}\right\} P\left(\left\{\boldsymbol{\sigma}^{\prime \prime}\right\}\right)\left[\int \mathrm{d} \mathbf{U} P(\mathbf{U}) \mathrm{e}^{\beta J \sum_{\alpha} \boldsymbol{\sigma}_{\alpha} \cdot \mathbf{U} \boldsymbol{\sigma}_{\alpha}^{\prime \prime}}-1\right]-\beta \sum_{\alpha} V\left(\boldsymbol{\sigma}_{\alpha}^{\prime}\right)\right)} \\
\bar{f}=\lim _{n \rightarrow 0} \frac{1}{\beta n}\left\{\frac{1}{2} c \int\left\{\mathrm{d} \boldsymbol{\sigma} \mathrm{d} \boldsymbol{\sigma}^{\prime}\right\} P(\{\boldsymbol{\sigma}\}) P\left(\left\{\boldsymbol{\sigma}^{\prime}\right\}\right)\left[\int \mathrm{d} \mathbf{U} P(\mathbf{U}) \exp \left(\beta J \sum_{\alpha} \boldsymbol{\sigma}_{\alpha} \cdot \mathbf{U} \boldsymbol{\sigma}_{\alpha}^{\prime}\right)-1\right]\right. \\
-\log \int\{\mathrm{d} \boldsymbol{\sigma}\} \exp \left(c \int\left\{\mathrm{d} \boldsymbol{\sigma}^{\prime}\right\} P\left(\left\{\boldsymbol{\sigma}^{\prime}\right\}\right)\right. \\
\left.\left.\times\left[\int \mathrm{d} \mathbf{U} P(\mathbf{U}) \mathrm{e}^{\beta J \sum_{\alpha} \sigma_{\alpha} \cdot \mathbf{U} \boldsymbol{\sigma}_{\alpha}^{\prime}}-1\right]-\beta \sum_{\alpha} V\left(\boldsymbol{\sigma}_{\alpha}\right)\right)\right\} .
\end{gathered}
$$

\subsection{Replica-symmetric theory}

We now make the canonical RS ansatz for continuous spins in our saddle-point equations ${ }^{7}$. We assume there to be a complete family of distributions $P[\sigma \mid \mu]$ on $S_{d-1}$, parametrized by a countable set of real-valued parameters $\boldsymbol{\mu}=\left(\mu_{0}, \mu_{1}, \mu_{2}, \ldots\right)$, such that

$$
P_{\mathrm{RS}}\left(\boldsymbol{\sigma}^{1}, \ldots, \boldsymbol{\sigma}_{n}\right)=\int \mathrm{d} \boldsymbol{\mu} w(\boldsymbol{\mu}) \prod_{\alpha} P\left[\boldsymbol{\sigma}_{\alpha} \mid \boldsymbol{\mu}\right]
$$

with a normalized density $w(\boldsymbol{\mu})$. A representation-independent but mathematically equivalent formulation of the RS ansatz follows upon defining the functional measure

$$
W[\{P\}]=\int \mathrm{d} \boldsymbol{\mu} w(\boldsymbol{\mu}) \prod_{\sigma \in S_{d-1}} \delta[P(\boldsymbol{\sigma})-P[\boldsymbol{\sigma} \mid \boldsymbol{\mu}]] .
$$

Here we used the symbolic notation $\prod_{\sigma} \delta[P(\sigma)-L(\sigma)]$ for the functional version of the $\delta$-distribution, defined via the identity $\int\{\mathrm{d} P\} G[\{P\}] \prod_{\sigma} \delta[P(\sigma)-L(\sigma)]=G[\{L\}]$. For

\footnotetext{
7 This ansatz reflects the complicating fact that, in the case of continuous spins, the RS order parameter function depends on the replicated spin variables not only via the sum $\sum_{\alpha} \sigma_{\alpha}$ (as would have been the case for Ising spins), but rather on all possible sums of the form $\sum_{\alpha} \sigma_{\alpha}^{K}$, for any $K \geqslant 1$.
} 
non-degenerate parametrizations, i.e., for those such that every function $P(\sigma)$ corresponds to a unique choice $\boldsymbol{\mu}(\{P\})$ of parameters, with $P[\boldsymbol{\sigma} \mid \boldsymbol{\mu}(\{P\})]=P(\sigma)$ for all $\boldsymbol{\sigma} \in S_{d-1}$, we can invert relation (10) and write

$$
w(\boldsymbol{\mu})=\int\{\mathrm{d} P\} W[\{P\}] \delta[\boldsymbol{\mu}-\boldsymbol{\mu}(\{P\})] .
$$

In terms of the functional measure $W[\{P\}]$ our RS ansatz (9) takes an elegant and representation-free form:

$$
P_{\mathrm{RS}}\left(\sigma^{1}, \ldots, \sigma_{n}\right)=\int\{\mathrm{d} P\} W[\{P\}] \prod_{\alpha} P\left(\sigma_{\alpha}\right) .
$$

The physical interpretation of our subsequent observables and results in terms of the original disordered $N$-spin system will follow from the identity

$$
\int\{\mathrm{d} P\} W[\{P\}] \prod_{\alpha}\left[\int \mathrm{d} \boldsymbol{\sigma} P(\boldsymbol{\sigma}) f_{\alpha}(\boldsymbol{\sigma})\right]=\lim _{N \rightarrow \infty} \frac{1}{N} \sum_{i} \overline{\prod_{\alpha}\left\langle f_{\alpha}\left(\sigma_{i}\right)\right\rangle} .
$$

We insert into our general saddle-point equation (7) the RS ansatz (12), and introduce the convention $\prod_{k=1}^{0} a_{k}=1$ for any series $\left\{a_{k}\right\}$. This leads to the following identity (with a constant $C_{n}$ which will in due course be determined by normalization),

$$
\begin{aligned}
C_{n} \int\{\mathrm{d} P\} W[\{P\}] & \prod_{\alpha} P\left(\boldsymbol{\sigma}_{\alpha}\right)=\exp \left(c \int\{\mathrm{d} P\} W[\{P\}] \int \mathrm{d} \mathbf{U} P(\mathbf{U})\right. \\
& \times \prod_{\alpha}\left[\int \mathrm{d} \boldsymbol{\sigma}^{\prime} P\left(\boldsymbol{\sigma}^{\prime}\right) \mathrm{e}^{\left.\beta J \sigma_{\alpha} \cdot \mathrm{U} \boldsymbol{\sigma}^{\prime}\right]}-c-\beta \sum_{\alpha} V\left(\boldsymbol{\sigma}_{\alpha}\right)\right) \\
= & \sum_{\ell \geqslant 0} \frac{c^{\ell}}{\ell !} \mathrm{e}^{-c} \int \prod_{k=1}^{\ell}\left[\left\{\mathrm{d} P_{k}\right\} W\left[\left\{P_{k}\right\}\right] \mathrm{d} \mathbf{U}_{k} P\left(\mathbf{U}_{k}\right)\right] \\
& \times \prod_{\alpha}\left\{\mathrm{e}^{-\beta V\left(\boldsymbol{\sigma}_{\alpha}\right)} \prod_{k=1}^{\ell} \int \mathrm{d} \boldsymbol{\sigma}^{\prime} P_{k}\left(\boldsymbol{\sigma}^{\prime}\right) \mathrm{e}^{\beta J \sigma_{\alpha} \cdot \mathbf{U}_{k} \boldsymbol{\sigma}^{\prime}}\right\} \\
= & \int\{\mathrm{d} P\} \prod_{\alpha} P\left(\boldsymbol{\sigma}_{\alpha}\right) \sum_{\ell \geqslant 0} \frac{c^{\ell}}{\ell !} \mathrm{e}^{-c} \int \prod_{k=1}^{\ell}\left[\left\{\mathrm{d} P_{k}\right\} W\left[\left\{P_{k}\right\}\right] \mathrm{d} \mathbf{U}_{k} P\left(\mathbf{U}_{k}\right)\right] Z^{n}\left[\left\{P_{1}, \ldots, P_{\ell}\right\}\right] \\
& \times \prod_{\boldsymbol{\sigma} \in S_{d} \delta} \delta\left[(\boldsymbol{\sigma})-\frac{\mathrm{e}^{-\beta V(\boldsymbol{\sigma})} \prod_{k=1}^{\ell} \int \mathrm{d} \boldsymbol{\sigma}^{\prime} P_{k}\left(\boldsymbol{\sigma}^{\prime}\right) \mathrm{e}^{\beta J \sigma \cdot \mathbf{U}_{k} \boldsymbol{\sigma}^{\prime}}}{Z\left[\left\{P_{1}, \ldots, P_{\ell}\right\}\right]}\right]
\end{aligned}
$$

where

$$
Z\left[\left\{P_{1}, \ldots, P_{\ell}\right\}\right]=\int \mathrm{d} \boldsymbol{\sigma} \mathrm{e}^{-\beta V(\boldsymbol{\sigma})} \prod_{k=1}^{\ell} \int \mathrm{d} \boldsymbol{\sigma}^{\prime} P_{k}\left(\boldsymbol{\sigma}^{\prime}\right) \mathrm{e}^{\beta J \boldsymbol{\sigma} \cdot \mathbf{U}_{k} \boldsymbol{\sigma}^{\prime}} .
$$

In the replica limit $n \rightarrow 0$, both the term $Z^{n}\left[\left\{P_{1}, \ldots, P_{k}\right\}\right]$ and the constant $C_{n}$ reduce to unity, and our RS order parameter equation acquires the transparent form

$$
\begin{aligned}
& W[\{P\}]=\sum_{\ell \geqslant 0} \frac{c^{\ell}}{\ell !} \mathrm{e}^{-c} \int \prod_{k \leqslant \ell}\left[\left\{\mathrm{d} P_{k}\right\} W\left[\left\{P_{k}\right\}\right] \mathrm{d} \mathbf{U}_{k} P\left(\mathbf{U}_{k}\right)\right] \\
& \times \prod_{\sigma \in S_{d-1}} \delta\left[P(\boldsymbol{\sigma})-\frac{\mathrm{e}^{-\beta V(\boldsymbol{\sigma})} \prod_{k=1}^{\ell} \int \mathrm{d} \boldsymbol{\sigma}^{\prime} P_{k}\left(\boldsymbol{\sigma}^{\prime}\right) \mathrm{e}^{\beta J \boldsymbol{\sigma} \cdot \mathbf{U}_{k} \boldsymbol{\sigma}^{\prime}}}{\left.\int \mathrm{d} \boldsymbol{\sigma}^{\prime \prime} \mathrm{e}^{-\beta V\left(\boldsymbol{\sigma}^{\prime \prime}\right)} \prod_{k=1}^{\ell} \int \mathrm{d} \boldsymbol{\sigma}^{\prime} P_{k}\left(\boldsymbol{\sigma}^{\prime}\right) \mathrm{e}^{\beta J \sigma^{\prime \prime} \cdot \mathbf{U}_{k} \boldsymbol{\sigma}^{\prime}}\right] .}\right.
\end{aligned}
$$


The replica-symmetric order parameter for finitely and randomly connected disordered systems with continuous degrees of freedom is thus seen to be a functional $W[\{P\}]$ on the space of probability densities. For any specific parametrization $P[\boldsymbol{\sigma} \mid \boldsymbol{\mu}]$ of this space, the order parameter equation (16) becomes an equation for the distribution $w(\boldsymbol{\mu})$ of parameters (of which there must generally be an infinite number):

$$
\begin{aligned}
& w(\boldsymbol{\mu})=\sum_{\ell \geqslant 0} \frac{c^{\ell}}{\ell !} \mathrm{e}^{-c} \int \prod_{k \leqslant \ell}\left[\mathrm{d} \boldsymbol{\mu}_{k} w\left(\boldsymbol{\mu}_{k}\right) \mathrm{d} \mathbf{U}_{k} P\left(\mathbf{U}_{k}\right)\right] \int\{\mathrm{d} P\} \delta[\boldsymbol{\mu}-\boldsymbol{\mu}(\{P\})] \\
& \times \prod_{\boldsymbol{\sigma} \in S_{d-1}} \delta\left[P(\boldsymbol{\sigma})-\frac{\mathrm{e}^{-\beta V(\boldsymbol{\sigma})} \prod_{k=1}^{\ell} \int \mathrm{d} \boldsymbol{\sigma}^{\prime} P\left[\boldsymbol{\sigma}^{\prime} \mid \boldsymbol{\mu}_{k}\right] \mathrm{e}^{\beta J \boldsymbol{\sigma} \cdot \mathbf{U}_{k} \boldsymbol{\sigma}^{\prime}}}{\left.\int \mathrm{d} \boldsymbol{\sigma}^{\prime \prime} \mathrm{e}^{-\beta V\left(\boldsymbol{\sigma}^{\prime \prime}\right)} \prod_{k=1}^{\ell} \int \mathrm{d} \boldsymbol{\sigma}^{\prime} P\left[\boldsymbol{\sigma}^{\prime} \mid \boldsymbol{\mu}_{k}\right] \mathrm{e}^{\beta J \boldsymbol{\sigma}^{\prime \prime} \cdot \mathbf{U}_{k} \boldsymbol{\sigma}^{\prime}}\right] .}\right.
\end{aligned}
$$

We note that multiplication of $P[\sigma \mid \boldsymbol{\mu}]$ by a constant will not affect (17), so that in our parametrizations $P[\boldsymbol{\sigma} \mid \boldsymbol{\mu}]$ we need not impose normalization explicitly. Application of the above manipulations to formula (8) leads us in a similar manner to the following expression for the RS free energy:

$$
\begin{aligned}
\bar{f}_{\mathrm{RS}}=\frac{c}{2 \beta} \int\left\{\mathrm{d} P_{1} \mathrm{~d} P_{2}\right\} W\left[\left\{P_{1}\right\}\right] W\left[\left\{P_{2}\right\}\right] \int \mathrm{d} \mathbf{U} P(\mathbf{U}) \log \left[\int \mathrm{d} \boldsymbol{\sigma} \mathrm{d} \boldsymbol{\sigma}^{\prime} P_{1}(\boldsymbol{\sigma}) P_{2}\left(\boldsymbol{\sigma}^{\prime}\right) \mathrm{e}^{\beta J \boldsymbol{\sigma} \cdot \mathbf{U} \boldsymbol{\sigma}^{\prime}}\right] \\
\quad-\frac{1}{\beta} \sum_{\ell \geqslant 0} \frac{c^{\ell}}{\ell !} \mathrm{e}^{-c} \int \prod_{k=1}^{\ell}\left[\left\{\mathrm{d} P_{k}\right\} W\left[\left\{P_{k}\right\}\right] \mathrm{d} \mathbf{U}_{k} P\left(\mathbf{U}_{k}\right)\right] \\
\times \log \left\{\int \mathrm{d} \boldsymbol{\sigma} \mathrm{e}^{-\beta V(\sigma)} \prod_{k=1}^{\ell} \int \mathrm{d} \boldsymbol{\sigma}^{\prime} P_{k}\left(\boldsymbol{\sigma}^{\prime}\right) \mathrm{e}^{\beta J \sigma \cdot \mathbf{U}_{k} \boldsymbol{\sigma}^{\prime}}\right\}
\end{aligned}
$$

An alternative formalism for the mathematical analysis of finitely connected spin models and deriving the key equations (16) and (18) would have been the cavity method; see, e.g., [7] and [8]. Which route one prefers is largely a matter of taste or familiarity ${ }^{8}$.

\subsection{The Ising limit}

As a simple consistency test of our theory, we now consider the limit $\gamma \rightarrow \infty$ of our population dynamics equation (16), for the special choice $V(\boldsymbol{\sigma})=\gamma(\hat{\mathbf{e}} \cdot \boldsymbol{\sigma})^{2}$ where $\hat{\mathbf{e}}$ denotes an arbitrary unit-length vector in $\mathbb{R}^{d}$. Via a saddle-point argument, we observe that the limit $\gamma \rightarrow \infty$ restricts our spins to take one of two possible values, namely, $\sigma= \pm \hat{\mathbf{e}}$. Hence, our order parameter function $P(\sigma)$ collapses to a sum of two delta peaks, which (with a modest amount of foresight) we choose to parametrize by

$$
P(\boldsymbol{\sigma})=\frac{\mathrm{e}^{\beta h}}{2 \cosh (\beta h)} \delta(\boldsymbol{\sigma}-\hat{\mathbf{e}})+\frac{\mathrm{e}^{-\beta h}}{2 \cosh (\beta h)} \delta(\boldsymbol{\sigma}+\hat{\mathbf{e}}) .
$$

With this representation of $P(\sigma)$, our order parameter functional $W[\{P\}]$ reduces to a distribution $W(h)$ of 'effective' fields $h$. Carrying out the integrations within the $\delta$-functional in (16) then results in

\footnotetext{
8 Each method has its own advantages and disadvantages: the replica formalism is somewhat more easily generalized to include states with complex ergodicity breaking (RSB), whereas the cavity concept (where one does not average over random graphs) allows one to interpret equations such as (16) as a self-consistency equation for the spin probability density at a site where a bond has been removed, which (in turn) allows for immediate generalization of the theory to random graphs with arbitrary degree distributions.
} 


$$
\begin{aligned}
W(h)=\sum_{\ell \geqslant 0} \frac{\mathrm{e}^{-c} c^{\ell}}{\ell !} \int \prod_{k \leqslant \ell}\left[\mathrm{d} h_{k} W\left(h_{k}\right) \mathrm{d} \mathbf{U}_{k} P\left(\mathbf{U}_{k}\right)\right] \prod_{\sigma \in\{-\hat{\mathbf{e}}, \hat{\mathbf{e}}\}} \delta\left[\frac{\exp [\beta h(\sigma \cdot \hat{\mathbf{e}})]}{2 \cosh (\beta h)}\right. \\
\left.-\frac{\prod_{k=1}^{\ell}\left[\frac{\exp \left(\beta h_{k}+\beta J \sigma \cdot \mathbf{U}_{k} \hat{\mathbf{e}}\right)}{2 \cosh \left(\beta h_{k}\right)}+\frac{\exp \left(-\beta h_{k}-\beta J \sigma \cdot \mathbf{U}_{k} \hat{\mathbf{e}}\right)}{2 \cosh \left(\beta h_{k}\right)}\right]}{\sum_{\sigma^{\prime} \in\{-\hat{\mathbf{e}}, \hat{\mathbf{e}}\}} \prod_{k=1}^{\ell}\left[\frac{\exp \left(\beta h_{k}+\beta J \sigma^{\prime} \cdot \mathbf{U}_{k} \hat{\mathbf{e}}\right)}{2 \cosh \left(\beta h_{k}\right)}+\frac{\exp \left(-\beta h_{k}-\beta J \sigma^{\prime} \cdot \mathbf{U}_{k} \hat{\mathbf{e}}\right)}{2 \cosh \left(\beta h_{k}\right)}\right]}\right] .
\end{aligned}
$$

The two $\delta$-functions in this expression now effectively give us an update relation for $h$. This leads to a population dynamics equation, equivalent to that found in, e.g., [2, 3]:

$$
\begin{aligned}
W(h)=\sum_{\ell \geqslant 0} \frac{\mathrm{e}^{-c} c^{\ell}}{\ell !} \int \prod_{k \leqslant \ell}\left[\mathrm{d} h_{k} W\left(h_{k}\right) \mathrm{d} \mathbf{U}_{k} P\left(\mathbf{U}_{k}\right)\right] \\
\quad \times \delta\left[h-\frac{1}{\beta} \sum_{k=1}^{\ell} \operatorname{arctanh}\left[\tanh (\beta h) \tanh \left(\beta J \hat{\mathbf{e}} \cdot \mathbf{U}_{k} \hat{\mathbf{e}}\right)\right]\right] .
\end{aligned}
$$

Choosing, for instance, the random orthogonal matrix distribution $P(\mathbf{U})$ to be of the simple form $P(\mathbf{U})=a \delta[\mathbf{U}-\mathbb{1}]+(1-a) \delta[\mathbf{U}+\mathbb{1}]$, with $a \in[0,1],{ }^{9}$ leads directly to the familiar functional order parameter equations of the $\pm J$ Ising spin-glass, with exchange interactions distributed according to $P\left(J^{\prime}\right)=a \delta\left[J^{\prime}-J\right]+(1-a) \delta\left[J^{\prime}+J\right]$.

\section{General theory for $d=2$}

\subsection{RS saddle-point equations and free energy}

For $d=2$ the set $S_{d-1}$ reduces to the unit circle, and our spins become $X Y$ spins, i.e., $\boldsymbol{\sigma}=(\cos \phi, \sin \phi)$ with $\phi \in[0,2 \pi]$. We may therefore also write $V(\sigma)=\tilde{V}(\phi)$, and find the random rotation matrices $\mathbf{U}$ being simply characterized by a single angle $\omega \in[0,2 \pi]$ and an associated symmetric distribution $P(\omega)=P(-\omega)$. As a result our equations simplify considerably. The order parameter equation (16) reduces to

$$
\begin{aligned}
W[\{P\}]= & \sum_{\ell \geqslant 0} \frac{c^{\ell}}{\ell !} \mathrm{e}^{-c} \int \prod_{k \leqslant \ell}\left[\left\{\mathrm{d} P_{k}\right\} W\left[\left\{P_{k}\right\}\right] \mathrm{d} \omega_{k} P\left(\omega_{k}\right)\right] \\
& \times \prod_{\phi} \delta\left[P(\phi)-\frac{\mathrm{e}^{-\beta \tilde{V}(\phi)} \prod_{k=1}^{\ell} \int \mathrm{d} \phi^{\prime} P_{k}\left(\phi^{\prime}\right) \exp \left(\beta J \cos \left(\phi-\phi^{\prime}-\omega_{k}\right)\right)}{\int \mathrm{d} \phi^{\prime \prime} \mathrm{e}^{-\beta \tilde{V}\left(\phi^{\prime \prime}\right)} \prod_{k=1}^{\ell} \int \mathrm{d} \phi^{\prime} P_{k}\left(\phi^{\prime}\right) \exp \left(\beta J \cos \left(\phi^{\prime \prime}-\phi^{\prime}-\omega_{k}\right)\right)}\right]
\end{aligned}
$$

In the absence of single-site potentials, i.e., for $\tilde{V}(\phi)=0$, we get

$$
\begin{aligned}
W[\{P\}]= & \sum_{\ell \geqslant 0} \frac{c^{\ell}}{\ell !} \mathrm{e}^{-c} \int \prod_{k \leqslant \ell}\left[\left\{\mathrm{d} P_{k}\right\} W\left[\left\{P_{k}\right\}\right] \mathrm{d} \omega_{k} P\left(\omega_{k}\right)\right] \\
& \times \prod_{\phi} \delta\left[P(\phi)-\frac{\prod_{k=1}^{\ell} \int \mathrm{d} \phi^{\prime} P_{k}\left(\phi^{\prime}\right) \exp \left(\beta J \cos \left(\phi-\phi^{\prime}-\omega_{k}\right)\right)}{\int \mathrm{d} \phi^{\prime \prime} \prod_{k=1}^{\ell} \int \mathrm{d} \phi^{\prime} P_{k}\left(\phi^{\prime}\right) \exp \left(\beta J \cos \left(\phi^{\prime \prime}-\phi^{\prime}-\omega_{k}\right)\right)}\right] .
\end{aligned}
$$

\footnotetext{
9 Here we take the freedom to consider the orthogonal group $O(3)$ instead of $S O(3)$, otherwise the matrix $-\mathbb{1}$ would not be in our ensemble (defined as the orthogonal matrices with determinant 1).
} 
For $T=0$ this reduces even further to

$$
\begin{aligned}
W[\{P\}]= & \sum_{\ell \geqslant 0} \frac{c^{\ell}}{\ell !} \mathrm{e}^{-c} \int \prod_{k \leqslant \ell}\left[\left\{\mathrm{d} P_{k}\right\} W\left[\left\{P_{k}\right\}\right] \mathrm{d} \omega_{k} P\left(\omega_{k}\right)\right] \\
& \times \prod_{\phi} \delta\left[P(\phi)-\frac{\prod_{k=1}^{\ell} P_{k}\left(\phi-\omega_{k}\right)}{\int \mathrm{d} \phi^{\prime \prime} \prod_{k=1}^{\ell} P_{k}\left(\phi^{\prime \prime}-\omega_{k}\right)}\right] .
\end{aligned}
$$

Whenever we carry out angle shifts such as those used in deriving (24), we take the distributions $P_{k}(\phi)$ to be continued periodically outside the domain $[0,2 \pi]$. Similarly, we find that for $V(\sigma)=0$ and $d=2$ the RS free energy per spin (18) becomes

$$
\begin{aligned}
\bar{f}_{\mathrm{RS}}=\frac{c}{2 \beta} \int\left\{\mathrm{d} P_{1} \mathrm{~d} P_{2}\right\} W\left[\left\{P_{1}\right\}\right] W\left[\left\{P_{2}\right\}\right] \int \mathrm{d} \omega P(\omega) \\
\quad \times \log \left[\int \mathrm{d} \phi \mathrm{d} \phi^{\prime} P_{1}(\phi) P_{2}\left(\phi^{\prime}\right) \exp \left(\beta J \cos \left(\phi-\phi^{\prime}-\omega\right)\right)\right] \\
-\frac{1}{\beta} \sum_{\ell \geqslant 0} \frac{c^{\ell}}{\ell !} \mathrm{e}^{-c} \int \prod_{k=1}^{\ell}\left[\left\{\mathrm{d} P_{k}\right\} W\left[\left\{P_{k}\right\}\right] \mathrm{d} \omega_{k} P\left(\omega_{k}\right)\right] \\
\times \log \left[\int \mathrm{d} \phi \prod_{k=1}^{\ell} \int \mathrm{d} \phi^{\prime} P_{k}\left(\phi^{\prime}\right) \exp \left(\beta J \cos \left(\phi-\phi^{\prime}-\omega_{k}\right)\right)\right] .
\end{aligned}
$$

As expected, the paramagnetic state $W[\{P\}]=\prod_{\phi \in[0,2 \pi]} \delta\left[P(\phi)-(2 \pi)^{-1}\right]$ is a solution of (23) at any temperature. In this state one finds, using (13), that $\left\langle\delta\left[\phi-\phi_{i}\right]\right\rangle=(2 \pi)^{-1}$ for all $i$ and all $\phi$.

Continuous bifurcations away from the paramagnetic state can be identified via a socalled Guzai (or functional moment) expansion. We transform $P(\phi) \rightarrow(2 \pi)^{-1}+\Delta(\phi)$, with $W[\{P\}] \rightarrow \tilde{W}[\{\Delta\}]$ and with $\tilde{W}[\{\Delta\}]=0$ as soon as $\int_{0}^{2 \pi} \mathrm{d} \phi \Delta(\phi) \neq 0$ (since $P(\phi)$ must remain normalized). We may now expand our equations in powers of the functional moments $\int\{\mathrm{d} \Delta\} \tilde{W}[\{\Delta\}] \Delta\left(\phi_{1}\right) \ldots \Delta\left(\phi_{r}\right)$ for $r=1,2$. In doing so we will repeatedly encounter the modified Bessel functions

$$
I_{n}(z)=\int_{-\pi}^{\pi} \frac{\mathrm{d} \phi}{2 \pi} \cos (n \phi) \mathrm{e}^{z \cos (\phi)} .
$$

Close to a continuous phase transition we assume there to be a small parameter $\epsilon$ measuring the bifurcation, such that $\int\{\mathrm{d} \Delta\} \tilde{W}[\{\Delta\}] \Delta\left(\phi_{1}\right) \ldots \Delta\left(\phi_{r}\right)=\mathcal{O}\left(\epsilon^{r}\right)$. Any bifurcations that this procedure identifies represent possible physical instabilities of the paramagnetic state, in the absence of external fields, towards the formation of spontaneously maintained non-uniform orientation statistics of individual spins.

\subsection{Paramagnetic to ferromagnetic and Kosterlitz-Thouless-type transitions}

We first inspect the lowest order, i.e., $\epsilon^{1}$, bifurcations away from the paramagnetic solution of the $\tilde{V}(\phi)=0$ equation (23). Here we have

$$
\begin{array}{r}
\int\{\mathrm{d} \Delta\} \tilde{W}[\{\Delta\}] \Delta(\phi)=\frac{1}{2 \pi} \sum_{\ell \geqslant 0} \frac{c^{\ell}}{\ell !} \mathrm{e}^{-c} \int \prod_{k \leqslant \ell}\left[\left\{\mathrm{d} \Delta_{k}\right\} \tilde{W}\left[\left\{\Delta_{k}\right\}\right] \mathrm{d} \omega_{k} P\left(\omega_{k}\right)\right] \\
\times\left\{\frac{1+\sum_{k=1}^{\ell} \frac{\int \mathrm{d} \phi^{\prime} \Delta_{k}\left(\phi^{\prime}\right) \exp \left(\beta J \cos \left(\phi-\phi^{\prime}-\omega_{k}\right)\right)}{I_{0}(\beta J)}+\cdots}{1+\sum_{k=1}^{\ell} \int \frac{\mathrm{d} \phi^{\prime \prime}}{2 \pi} \frac{\int \mathrm{d} \phi^{\prime} \Delta_{k}\left(\phi^{\prime}\right) \exp \left(\beta J \cos \left(\phi^{\prime \prime}-\phi^{\prime}-\omega_{k}\right)\right)}{I_{0}(\beta J)}+\cdots}-1\right\}
\end{array}
$$




$$
\begin{aligned}
= & \frac{1}{2 \pi} \sum_{\ell \geqslant 0} \frac{c^{\ell}}{\ell !} \mathrm{e}^{-c} \int \prod_{k \leqslant \ell}\left[\left\{\mathrm{d} \Delta_{k}\right\} \tilde{W}\left[\left\{\Delta_{k}\right\}\right] \mathrm{d} \omega_{k} P\left(\omega_{k}\right)\right] \\
& \times \sum_{k=1}^{\ell}\left\{\frac{\int \mathrm{d} \phi^{\prime} \Delta_{k}\left(\phi^{\prime}\right) \exp \left(\beta J \cos \left(\phi-\phi^{\prime}-\omega_{k}\right)\right)}{I_{0}(\beta J)}\right. \\
& \left.-\int \frac{\mathrm{d} \phi^{\prime \prime}}{2 \pi} \frac{\int \mathrm{d} \phi^{\prime} \Delta_{k}\left(\phi^{\prime}\right) \exp \left(\beta J \cos \left(\phi^{\prime \prime}-\phi^{\prime}-\omega_{k}\right)\right)}{I_{0}(\beta J)}+\cdots\right\} \\
= & \frac{c}{2 \pi I_{0}(\beta J)} \\
& \times \int\{\mathrm{d} \Delta\} \tilde{W}[\{\Delta\}] \mathrm{d} \omega P(\omega) \int \mathrm{d} \phi^{\prime} \Delta\left(\phi^{\prime}\right) \exp \left(\beta J \cos \left(\phi-\phi^{\prime}-\omega\right)\right)+\mathcal{O}\left(\epsilon^{2}\right) .
\end{aligned}
$$

Thus, with $\Psi(\phi)=\int\{\mathrm{d} \Delta\} \tilde{W}[\{\Delta\}] \Delta(\phi)$ we obtain the following (constrained) leading order eigenvalue problem, which describes transitions away from the paramagnetic state:

$$
\begin{aligned}
& \Psi(\phi)=\frac{c}{2 \pi I_{0}(\beta J)} \int_{0}^{2 \pi} \mathrm{d} \phi^{\prime} \int \mathrm{d} \omega P(\omega) \exp \left(\beta J \cos \left(\phi-\phi^{\prime}-\omega\right)\right) \Psi\left(\phi^{\prime}\right) \\
& \int_{0}^{2 \pi} \mathrm{d} \phi \Psi(\phi)=0 .
\end{aligned}
$$

This problem is solved by the Fourier modes $\Psi(\phi)=\hat{\Psi}_{k} \mathrm{e}^{\mathrm{i} k \phi}$ (with integer $k \neq 0$ ), each of which bifurcates at a temperature $T_{k}$ which is to be solved from

$$
1=\frac{c I_{k}(\beta J)}{I_{0}(\beta J)} \int_{-\pi}^{\pi} \mathrm{d} \omega P(\omega) \cos (k \omega) .
$$

For either $\beta \rightarrow 0$ or $c \rightarrow 0$ the right-hand side would reduce to zero, and we would find ourselves always in a paramagnetic state. The presently studied transition therefore occurs at the average connectivity $c$ for which

$$
c=\min _{k>0}\left\{\frac{I_{k}(\beta J)}{I_{0}(\beta J)} \int_{-\pi}^{\pi} \mathrm{d} \omega P(\omega) \cos (k \omega)\right\}^{-1} .
$$

At zero temperature we may use the property $\lim _{z \rightarrow \infty} I_{k}(z) / I_{0}(z)=1$ to obtain $c_{\text {crit }}^{-1}=$ $\max _{k>0} \int_{-\pi}^{\pi} \mathrm{d} \omega P(\omega) \cos (k \omega) \leqslant 1$. This could have been expected, since the percolation transition in the random graph (2) occurs at $c=1$. According to (13), the present type of bifurcation is towards a state where (with $k$ denoting the critical Fourier mode)

$$
\lim _{N \rightarrow \infty} \frac{1}{N} \sum_{i} \overline{\left\langle\delta\left[\phi-\phi_{i}\right]\right\rangle}=\frac{1}{2 \pi}[1+\epsilon \cos (k \phi-\psi)+\cdots] .
$$

Care is to be taken in interpreting the bifurcating state, since in leading order one has

$$
\begin{aligned}
\lim _{N \rightarrow \infty} \frac{1}{N} \sum_{i} \overline{\left\langle\sigma_{i}\right\rangle} & =\lim _{N \rightarrow \infty} \frac{1}{N} \sum_{i} \overline{\left\langle\left(\begin{array}{c}
\cos \left(\phi_{i}\right) \\
\sin \left(\phi_{i}\right)
\end{array}\right)\right\rangle} \\
& =\frac{\epsilon}{2 \pi}\left(\begin{array}{c}
\int_{-\pi}^{\pi} \mathrm{d} \phi \cos (\phi) \cos (k \phi-\psi) \\
\int_{-\pi}^{\pi} \mathrm{d} \phi \sin (\phi) \cos (k \phi-\psi)
\end{array}\right)+\cdots \\
& =\frac{1}{2} \epsilon \delta_{k 1}\left(\begin{array}{c}
\cos (\psi) \\
\sin (\psi)
\end{array}\right)+\cdots
\end{aligned}
$$


We conclude that only for $k=1$ may we call the bifurcating solution ferromagnetic $(\mathrm{F})$. For $k>1$ we find a bifurcation towards a state with no overall magnetization, but still with measurably non-uniform overall single spin statistics. This transition is reminiscent of a Kosterlitz-Thouless one. Thus we have the following possible transitions:

$$
\begin{aligned}
& \mathrm{P} \rightarrow \mathrm{F}: \quad c=\left\{\frac{I_{1}(\beta J)}{I_{0}(\beta J)} \int_{-\pi}^{\pi} \mathrm{d} \omega P(\omega) \cos (\omega)\right\}^{-1} \\
& \mathrm{KT}: \quad c=\min _{k>1}\left\{\frac{I_{k}(\beta J)}{I_{0}(\beta J)} \int_{-\pi}^{\pi} \mathrm{d} \omega P(\omega) \cos (k \omega)\right\}^{-1} .
\end{aligned}
$$

It will be shown below, however, that the KT transition is always preceded by a spin-glass transition, and hence it is non-physical.

\subsection{Paramagnetic to spin-glass transition}

If the transition away from the paramagnetic solution of the $\tilde{V}(\phi)=0$ equation (23) is towards a new state with $\int\{\mathrm{d} \Delta\} \tilde{W}[\{\Delta\}] \Delta(\phi)=0$ (which would be a spin-glass state), the lowest relevant order in our expansions is $\epsilon^{2}$, and we find after functional moment expansion that

$$
\begin{aligned}
\int\{\mathrm{d} \Delta\} \tilde{W}[\{\Delta\}] & \Delta\left(\phi_{1}\right) \Delta\left(\phi_{2}\right)=\frac{c}{(2 \pi)^{2} I_{0}^{2}(\beta J)} \int[\{\mathrm{d} \Delta\} \tilde{W}[\{\Delta\}] \mathrm{d} \omega P(\omega)] \\
& \times\left(\int \mathrm{d} \phi^{\prime} \Delta\left(\phi^{\prime}\right) \exp \left(\beta J \cos \left(\phi_{1}-\phi^{\prime}-\omega\right)\right)\right) \\
& \times\left(\int \mathrm{d} \phi^{\prime} \Delta\left(\phi^{\prime}\right) \exp \left(\beta J \cos \left(\phi_{2}-\phi^{\prime}-\omega\right)\right)\right)+\mathcal{O}\left(\epsilon^{3}\right)
\end{aligned}
$$

Thus, with $\Psi\left(\phi_{1}, \phi_{2}\right)=\int\{\mathrm{d} \Delta\} \tilde{W}[\{\Delta\}] \Delta\left(\phi_{1}\right) \Delta\left(\phi_{2}\right)$ we now arrive at the following (constrained) eigenvalue problem for the $\mathrm{P} \rightarrow \mathrm{SG}$ transition:

$$
\begin{gathered}
\Psi\left(\phi_{1}, \phi_{2}\right)=c \int \frac{\mathrm{d} \phi_{1}^{\prime} \mathrm{d} \phi_{2}^{\prime}}{\left[2 \pi I_{0}(\beta J)\right]^{2}}\left[\int \mathrm { d } \omega P ( \omega ) \operatorname { e x p } \left(\beta J \cos \left(\phi_{1}-\phi_{1}^{\prime}-\omega\right)\right.\right. \\
\left.\left.+\beta J \cos \left(\phi_{2}-\phi_{2}^{\prime}-\omega\right)\right)\right] \Psi\left(\phi_{1}^{\prime}, \phi_{2}^{\prime}\right) \\
\int \mathrm{d} \phi_{1} \Psi\left(\phi_{1}, \phi_{2}\right)=\int \mathrm{d} \phi_{2} \Psi\left(\phi_{1}, \phi_{2}\right)=0 .
\end{gathered}
$$

Again one finds that the Fourier modes are the relevant solutions. Here we have $\Psi\left(\phi_{1}, \phi_{2}\right)=$ $\hat{\Psi}_{k_{1}, k_{2}} \mathrm{e}^{\mathrm{i}\left(k_{1} \phi_{1}+k_{2} \phi_{2}\right)}$ (with integer $k_{r} \neq 0$ ), each of which bifurcates at a temperature (or, equivalently, at a critical connectivity) which is to be solved from

$$
1=\frac{c I_{k_{1}}(\beta J) I_{k_{2}}(\beta J)}{I_{0}^{2}(\beta J)} \int \mathrm{d} \omega P(\omega) \cos \left[\left(k_{1}+k_{2}\right) \omega\right] .
$$

As before the right-hand side becomes zero for $\beta=0$ or $c \rightarrow 0$, so that the transition occurs at

$$
c=\min _{k_{1} \neq 0, k_{2} \neq 0}\left\{\frac{I_{k_{1}}(\beta J) I_{k_{2}}(\beta J)}{I_{0}^{2}(\beta J)} \int \mathrm{d} \omega P(\omega) \cos \left[\left(k_{1}+k_{2}\right) \omega\right]\right\}^{-1} .
$$

Since one always has $I_{k}(\beta J) \geqslant 0$ and $I_{-k}(z)=I_{k}(z)$, the required extremum occurs for $k_{1}=-k_{2}=k$. Hence we find $c=\min _{k>0} I_{0}^{2}(\beta J) / I_{k}^{2}(\beta J)$. Finally, one may 
use the general identity $I_{k+2}(z)=I_{k}(z)-2(k+1) I_{k+1}(z) / z$ (see, e.g., [42]) to infer that $\max _{k>0} I_{k}(\beta J)=\max \left\{I_{1}(\beta J), I_{2}(\beta J)\right\}=I_{1}(\beta J)$. We therefore conclude that

$$
\mathrm{P} \rightarrow \mathrm{SG}: \quad c=I_{0}^{2}(\beta J) / I_{1}^{2}(\beta J) .
$$

This equation is obviously independent of the distribution of chiral interactions. At zero temperature we find $c_{\text {crit }}=1$. According to (13), the present type of bifurcation is towards a state where

$$
\lim _{N \rightarrow \infty} \frac{1}{N} \sum_{i} \overline{\left\langle\delta\left[\phi-\phi_{i}\right]\right\rangle\left\langle\delta\left[\phi^{\prime}-\phi_{i}\right]\right\rangle}=\frac{1}{(2 \pi)^{2}}\left[1+\epsilon \cos \left(\phi-\phi^{\prime}+\psi\right)+\cdots\right] .
$$

We note that this particular type of bifurcation obeys $\lim _{N \rightarrow \infty} N^{-1} \sum_{i} \overline{\left\langle\delta\left[\phi-\phi_{i}\right]\right\rangle}=$ $(2 \pi)^{-1}$, i.e., the absence of measurable overall non-uniform spin statistics. Nevertheless, $\lim _{N \rightarrow \infty} N^{-1} \sum_{i} \overline{\left\langle\sigma_{i}\right\rangle^{2}}>0$, so the bifurcating solution describes a spin-glass state (SG).

\subsection{Summary of transitions away from paramagnetic state and special limits}

We define in accordance with the results (34), (41):

$$
\begin{aligned}
& c_{\mathrm{F}}^{-1}=\frac{I_{1}(\beta J)}{I_{0}(\beta J)} \int_{-\pi}^{\pi} \mathrm{d} \omega P(\omega) \cos (\omega) \\
& c_{\mathrm{SG}}^{-1}=I_{1}^{2}(\beta J) / I_{0}^{2}(\beta J) .
\end{aligned}
$$

The physical transition ${ }^{10}$ away from the paramagnetic state, as the connectivity is increased from $c=0$ for fixed $\beta J$, is the one with the largest value of $c_{\text {crit }}^{-1}$. The Kosterlitz-Thouless-type transition (35) was given by

$$
c_{\mathrm{KT}}^{-1}=\max _{k>1}\left\{\frac{I_{k}(\beta J)}{I_{0}(\beta J)} \int_{-\pi}^{\pi} \mathrm{d} \omega P(\omega) \cos (k \omega)\right\} \leqslant \frac{I_{2}(\beta J)}{I_{0}(\beta J)} .
$$

It follows from the properties of the modified Bessel functions [42] that $\frac{\mathrm{d}}{\mathrm{d} z}\left[I_{2}(z) I_{0}(z)-\right.$ $\left.I_{1}^{2}(z)\right]=\frac{1}{2} I_{0}(z)\left[I_{3}(z)-I_{0}(z)\right]<0$. Hence $I_{2}(z) / I_{0}(z)-I_{1}^{2}(z) / I_{0}^{2}(z) \leqslant 0$ with equality only for $z=0$. This implies that $c_{\mathrm{SG}}^{-1} \geqslant c_{\mathrm{KT}}^{-1}$ so that the bifurcation (35) is indeed unphysical. For small and large temperatures one obtains the limiting behaviour

$$
\begin{array}{lll}
\lim _{T \rightarrow 0} c_{\mathrm{F}}^{-1}=\int_{-\pi}^{\pi} \mathrm{d} \omega P(\omega) \cos (\omega) \leqslant 1 & \lim _{T \rightarrow \infty} c_{\mathrm{F}}^{-1}=0 \\
\lim _{T \rightarrow 0} c_{\mathrm{SG}}^{-1}=1 & \lim _{T \rightarrow \infty} c_{\mathrm{SG}}^{-1}=0 . &
\end{array}
$$

To recover known results in the $c \rightarrow \infty$ limit one must first rescale the bond strength $J$. In the case of an overall balance towards (anti-)ferromagnetism, i.e., for chirality distributions such that $\int_{-\pi}^{\pi} \mathrm{d} \omega P(\omega) \cos (\omega)=\mathcal{O}\left(c^{0}\right) \neq 0$, we have to rescale according to $J=\tilde{J} / c$. This gives upon taking $c \rightarrow \infty$, and using $I_{n}(z)=(z / 2)^{n} / n !+\mathcal{O}\left(z^{n+1}\right)$,

$$
T_{\mathrm{F}}=\frac{1}{2} \tilde{J} \int_{-\pi}^{\pi} \mathrm{d} \omega P(\omega) \cos (\omega) \quad(J=\tilde{J} / c, c \rightarrow \infty) .
$$

Here there is no transition towards an SG state ever. In the absence of such a dominant balance, i.e., for distributions such that $\int_{-\pi}^{\pi} \mathrm{d} \omega P(\omega) \cos (\omega)=\Lambda / \sqrt{c}$, the appropriate rescaling is $J=\tilde{J} / \sqrt{c}$. Now we find that

$$
T_{\mathrm{F}}=\frac{1}{2} \tilde{J} \Lambda \quad T_{\mathrm{SG}}=\frac{1}{2} \tilde{J} \quad(J=\tilde{J} / \sqrt{c}, c \rightarrow \infty) .
$$

${ }^{10}$ We will for now leave aside the possibility of first-order transitions. 


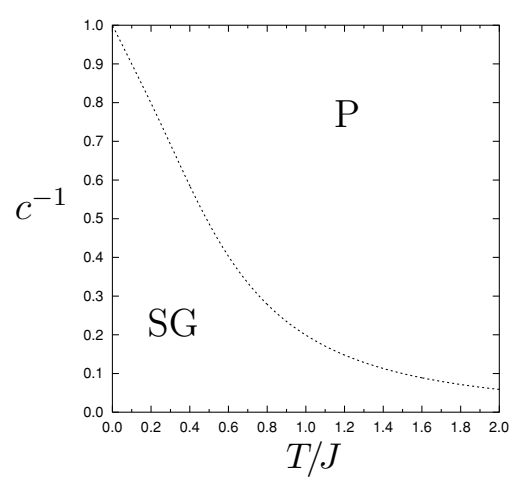

Figure 1. The predicted phase diagram for uniformly distributed chiralities $P(\omega)=(2 \pi)^{-1}$ and planar spins $(d=2)$. It describes a paramagnetic $(\mathrm{P})$ and a spin-glass (SG) phase, separated by a continuous phase transition (the dotted line).

Hence as $c \rightarrow \infty$ and the temperature is reduced from the paramagnetic state, for $\Lambda>1$ we will enter a ferromagnetic state, and for $\Lambda<1$ a spin-glass one. These results are in full agreement with those obtained earlier for fully connected systems; see, e.g., [39].

\section{Results for specific chirality distributions at $d=2$}

Let us now work out our transition lines (43), (44) for specific choices for the (symmetric) chirality distribution $P(\omega)$. It is clear from (44) that our choices will only affect the $\mathrm{P} \rightarrow$ $F$ transition. We note that so far we only have expressions for transitions away from the paramagnetic state; we are not yet able to determine the F $\rightarrow$ SG transition (when both F and SG phases exist) analytically, since this would require us to solve our equations below the $\mathrm{P} \rightarrow \mathrm{F}$ and/or $\mathrm{P} \rightarrow \mathrm{SG}$ transition temperatures. However, we may put forward the conjecture (on the basis of our experience with more conventional disordered spin models, e.g., [26, 40]) that, especially upon taking RSB into account (if needed), there will be no change of phase type after the onset of order as the temperature is lowered from $T=\infty$ to $T=0$. This conjecture would predict the elusive $\mathrm{F} \rightarrow \mathrm{SG}$ transition to be the horizontal line segment in the $\left(T, c^{-1}\right)$ phase diagram going from $T=0$ to the point where the $\mathrm{P} \rightarrow \mathrm{F}$ and $\mathrm{P} \rightarrow \mathrm{SG}$ lines meet (the Parisi-Toulouse hypothesis [41]).

\subsection{Predicted phase diagrams}

We first turn to uniformly distributed chiralities: $P(\omega)=(2 \pi)^{-1}$. Here we find that $c_{\mathrm{F}}^{-1}=0$ in (43), so for finite $c$ one only ever has the $\mathrm{P} \rightarrow \mathrm{SG}$ transition (44). The result of numerical evaluation of the latter bifurcation line is shown in figure 1.

Our second choice for the chirality statistics is the binary distribution $P(\omega)=\frac{1}{2} \delta(\omega-$ $\bar{\omega})+\frac{1}{2} \delta(\omega+\bar{\omega})$, with $\bar{\omega} \in[0, \pi]$. Here we find equations (43), (44) reducing to

$$
c_{\mathrm{F}}^{-1}=\frac{I_{1}(\beta J) \cos (\bar{\omega})}{I_{0}(\beta J)} \quad c_{\mathrm{SG}}^{-1}=\frac{I_{1}^{2}(\beta J)}{I_{0}^{2}(\beta J)} .
$$

Now both types of transitions are possible, and it will be clear that this can result in richer phase diagrams. The $\mathrm{P} \rightarrow \mathrm{F}$ transitions can only occur if $\bar{\omega} \in[0, \pi / 2]$; here, as $T \rightarrow 0$ we have $0 \leqslant \lim _{T \rightarrow 0} c_{\mathrm{F}}^{-1}=\cos (\bar{\omega}) \leqslant \lim _{T \rightarrow 0} c_{\mathrm{SG}}^{-1}=1$. For $\bar{\omega}=0$ (strictly ferromagnetic forces) one will only see a $\mathrm{P} \rightarrow \mathrm{F}$ transition. As we increase $\bar{\omega}$ away from the ferromagnetic value 


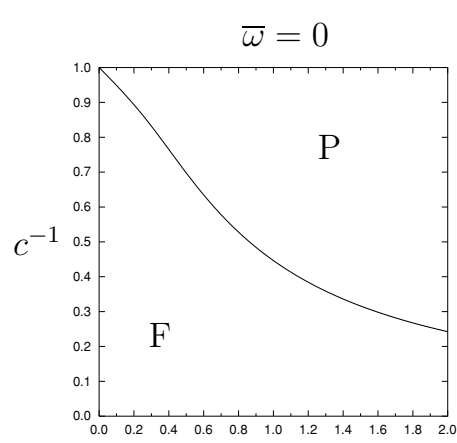

$T / J$

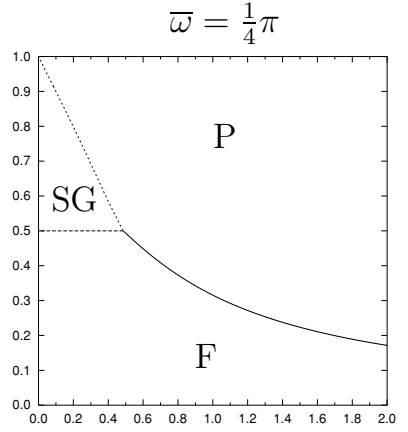

$T / J$

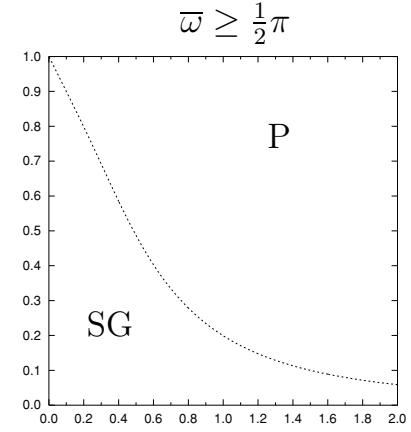

$T / J$

Figure 2. Continuous phase transitions away from the paramagnetic $(\mathrm{P})$ state for planar spins $(d=2)$ and binary chiralities $P(\omega)=\frac{1}{2} \delta(\omega-\bar{\omega})+\frac{1}{2} \delta(\omega+\bar{\omega})$. Solid lines: $\mathrm{P} \rightarrow \mathrm{F}$ bifurcations. Dotted lines: $\mathrm{P} \rightarrow \mathrm{SG}$ bifurcations. Note that the location of the $\mathrm{F} \rightarrow \mathrm{SG}$ transition (dashed) has not been calculated, but follows from the conjecture that on lowering temperature the nature of the ordered phase will remain that which emerges at the onset.
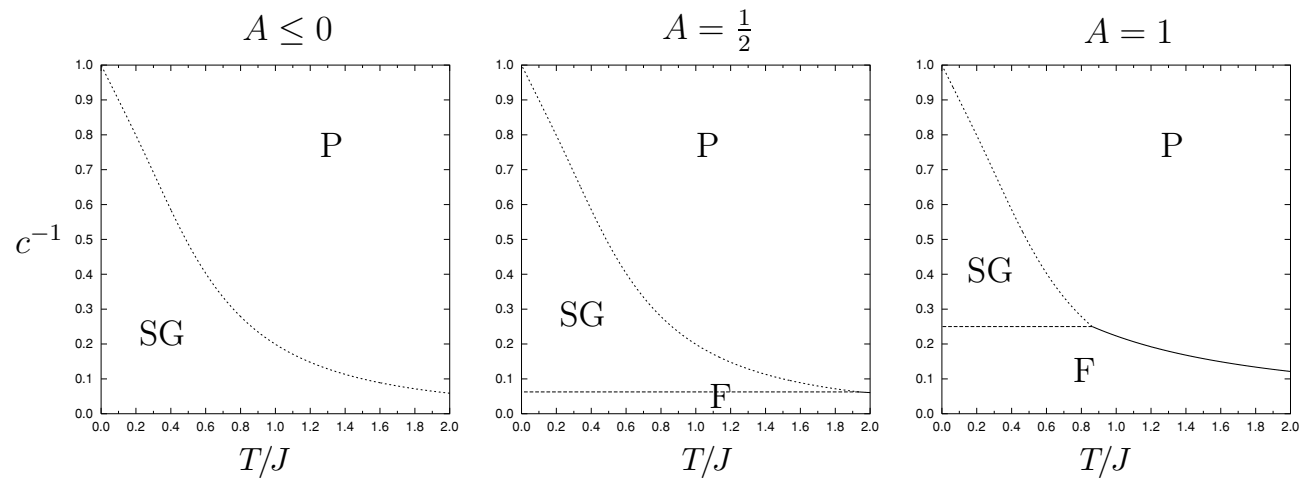

Figure 3. Continuous phase transitions away from the paramagnetic state for planar spins $(d=2)$ and resonant chiralities $P(\omega)=[1+A \cos (\ell \phi)] / 2 \pi$. Solid lines: $\mathrm{P} \rightarrow \mathrm{F}$ bifurcations. Dotted lines: $\mathrm{P} \rightarrow$ SG bifurcations. The location of the $\mathrm{F} \rightarrow \mathrm{SG}$ transition (dashed) has not been calculated, but follows from the conjecture that on lowering temperature the nature of the ordered phase will remain that which emerges at the onset.

$\bar{\omega}=0$ we see that at the point where $I_{1}(\beta J) / I_{0}(\beta J)=\cos (\bar{\omega})$ the $\mathrm{P} \rightarrow \mathrm{F}$ transition line crosses the $\mathrm{P} \rightarrow \mathrm{SG}$ one, until at $\bar{\omega} \geqslant \frac{1}{2} \pi$ the $\mathrm{F}$ phase has been completely eliminated. The result of numerical evaluation of the bifurcation lines (49) is shown in figure 2.

As a third example we inspect resonant chiralities: $P(\omega)=[1+A \cos (\ell \phi)] / 2 \pi$, with $A \in[-1,1]$ and $\ell \in\{1,2, \ldots\}$. Here we find, using $\int_{-\pi}^{\pi} \mathrm{d} \omega P(\omega) \cos (\omega)=\frac{1}{2} A \delta_{\ell 1}$, that for $A \leqslant 0$ and also all $\ell>1$ we only have the $\mathrm{P} \rightarrow \mathrm{SG}$ transition. If $A>0$ and $\ell=1$, on the other hand, we may enter either the F or the SG phase,

$$
\ell=1: \quad c_{\mathrm{F}}^{-1}=\frac{1}{2} A \frac{I_{1}(\beta J)}{I_{0}(\beta J)} \quad c_{\mathrm{SG}}^{-1}=\frac{I_{1}^{2}(\beta J)}{I_{0}^{2}(\beta J)},
$$

with a possible triple point for $I_{1}(\beta J) / I_{0}(\beta J)=\frac{1}{2} A$. The result of numerical evaluation of the bifurcation lines is shown in figure 3. Again we observe the competition between ferromagnetic and spin-glass order. 


\subsection{Numerical calculation of order parameters via population dynamics}

So far we have only shown results which did not involve solving our order parameter equations away from bifurcation points. We now probe our systems further by calculating observables (approximately) in the F and SG phases, and by comparing these with numerical simulations. Exact execution of this work programme would require us to solve the functional $W[\{P\}]$ from equation (16). In practice, one has to resort to explicit parametrizations $P[\boldsymbol{\sigma} \mid \boldsymbol{\mu}]$ of which the parameters $\boldsymbol{\mu}$ are truncated after a finite number of components, and solve instead the truncated version of (17). Here we choose

$$
P[\phi \mid \boldsymbol{\mu}]=\frac{\exp \left[\sum_{m \geqslant 1}\left(A_{m}^{c} \cos (m \phi)+A_{m}^{s} \sin (m \phi)\right)\right]}{\int \mathrm{d} \phi^{\prime} \exp \left[\sum_{m \geqslant 1}\left(A_{m}^{c} \cos \left(m \phi^{\prime}\right)+A_{m}^{s} \sin \left(m \phi^{\prime}\right)\right)\right]}
$$

with $\boldsymbol{\mu}=\left(A_{1}^{c}, A_{1}^{s}, A_{2}^{c}, A_{2}^{s}, \ldots\right)$. Using the orthogonality properties of $\cos (m \phi)$ and $\sin (m \phi)$ we can extract from (17) self-consistent equations for the measure $w(\boldsymbol{\mu})$. For the simplest case $\tilde{V}(\phi)=0$ these equations are found to take the form

$$
\begin{aligned}
w(\boldsymbol{\mu})= & \sum_{\ell \geqslant 0} \frac{\mathrm{e}^{-c} c^{\ell}}{\ell !} \int\left[\prod_{k \leqslant \ell} \mathrm{d} \boldsymbol{\mu}_{k} w\left(\boldsymbol{\mu}_{k}\right) \mathrm{d} \omega_{k} P\left(\omega_{k}\right)\right] \prod_{m \geqslant 1} \delta\left[A_{m}^{c}-\sum_{k \leqslant \ell} \int_{0}^{2 \pi} \frac{\mathrm{d} \phi}{\pi} \cos (m \phi)\right. \\
& \left.\times \log \int_{0}^{2 \pi} \mathrm{d} \phi^{\prime} \exp \left(\sum_{n>0} A_{n, k}^{c} \cos \left(n \phi^{\prime}\right)+A_{n, k}^{s} \sin \left(n \phi^{\prime}\right)+\beta J \cos \left(\phi-\phi^{\prime}-\omega_{k}\right)\right)\right] \\
& \times \prod_{m \geqslant 1} \delta\left[A_{m}^{s}-\sum_{k \leqslant \ell} \int_{0}^{2 \pi} \frac{\mathrm{d} \phi}{\pi} \sin (m \phi) \log \int_{0}^{2 \pi} \mathrm{d} \phi^{\prime}\right. \\
& \left.\times \exp \left(\sum_{n>0} A_{n, k}^{c} \cos \left(n \phi^{\prime}\right)+A_{n, k}^{s} \sin \left(n \phi^{\prime}\right)+\beta J \cos \left(\phi-\phi^{\prime}-\omega_{k}\right)\right)\right] .
\end{aligned}
$$

In order to solve these equations via, for example, the population dynamics ${ }^{11}$ scheme [7], one has to truncate the number of coefficients in the parametrization (51). In this paper, we have limited our analysis to a 2-coefficient truncation, i.e., $w(\boldsymbol{\mu}) \rightarrow w\left(A_{1}^{c}, A_{1}^{s}\right)$, and we have used populations of size $15 \times 10^{3}$ in the population dynamics algorithm. Increasing the order of the parametrization by small numbers was found to give only a modest improvement in accuracy, and it will turn out that already at the present level we find a good agreement between theory and (simulation) experiments.

The numerical (Monte Carlo) simulations, of which data are shown below, were carried out with systems of size $N=10^{5}$ and using the Fast Linear Algorithm of [45] (based on a sampling scheme that minimizes state rejections). The measurements (values of order parameters) were taken over $10^{5}$ iterations, following an equilibration stage of $10^{6}$ iterations. The only exception is figure $5(b)$, obtained using a simple first-order Euler method (i.e., the iteration of $\mathbf{y}_{n+1}=\mathbf{y}_{n}+h \mathbf{f}\left(\mathbf{y}_{n}, t_{n}\right)$ with $t_{n}=n t$ and small values of $h$, to approximate the solution of $\mathrm{d} \mathbf{y} / \mathrm{d} t=\mathbf{f}(\mathbf{y}, t))$; here we used elementary time steps of size $h=1 / 2 \mathrm{~N}$ and system size $N=1000$, after an equilibration stage of $4 \times 10^{3}$ iterations per spin.

\footnotetext{
${ }^{11}$ In this scheme, one exploits the mathematical consistency of interpreting equations such as (52) as the stationary state equations of a Markovian process for an evolving population of fields, with $w(\boldsymbol{\mu})$ representing the density of population members with a field of value $\boldsymbol{\mu}$. Equation (52) can thus be solved numerically, to a high accuracy, by simulation of this Markovian process.
} 


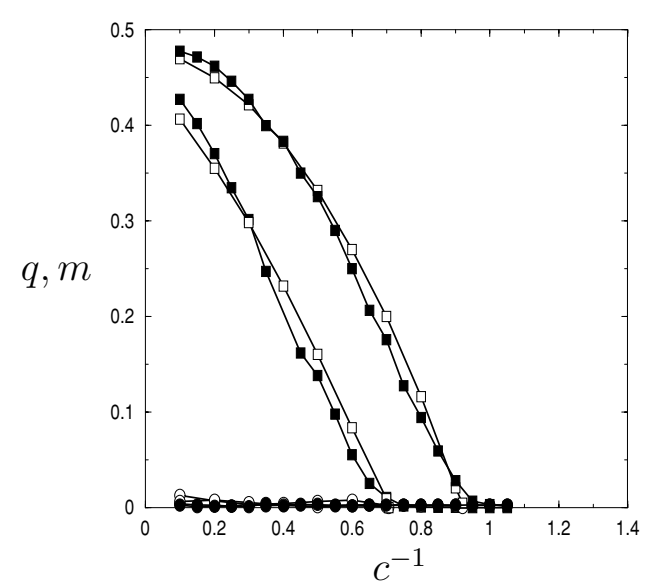

Figure 4. Population dynamics calculation of observables for uniformly distributed chiralities $P(\omega)=(2 \pi)^{-1}$ and planar spins $(d=2): q=\frac{1}{2}\left(q_{c c}+q_{s s}\right)$ (connected open squares) and $m=\sqrt{m_{c}^{2}+m_{s}^{2}}$ (connected open circles) as functions of $c^{-1}$, and for $T / J=0.1,0.3$ (upper versus lower curves). The observables were calculated with a population dynamics equation (52) and a truncated parametrization (see the text for details). Connected full squares and circles: corresponding simulation measurements of $q$ and $m$, for systems with $N=10^{5}$ spins.

In testing our theory against simulation experiments we focus mainly on the following four quantities:

$$
\begin{gathered}
m_{c}=\lim _{N \rightarrow \infty} \frac{1}{N} \sum_{i} \overline{\left\langle\cos \left(\phi_{i}\right)\right\rangle}=\int \mathrm{d} \boldsymbol{\mu} w(\boldsymbol{\mu}) \int \mathrm{d} \phi P[\phi \mid \boldsymbol{\mu}] \cos (\phi) \\
m_{s}=\lim _{N \rightarrow \infty} \frac{1}{N} \sum_{i} \overline{\left\langle\sin \left(\phi_{i}\right)\right\rangle}=\int \mathrm{d} \boldsymbol{\mu} w(\boldsymbol{\mu}) \int \mathrm{d} \phi P[\phi \mid \boldsymbol{\mu}] \sin (\phi) \\
q_{c c}=\lim _{N \rightarrow \infty} \frac{1}{N} \sum_{i} \overline{\left\langle\cos \left(\phi_{i}\right)\right\rangle^{2}}=\int \mathrm{d} \boldsymbol{\mu} w(\boldsymbol{\mu}) \int \mathrm{d} \phi \mathrm{d} \phi^{\prime} P[\phi \mid \boldsymbol{\mu}] P\left[\phi^{\prime} \mid \boldsymbol{\mu}\right] \cos (\phi) \cos \left(\phi^{\prime}\right) \\
q_{s s}=\lim _{N \rightarrow \infty} \frac{1}{N} \sum_{i} \overline{\left\langle\sin \left(\phi_{i}\right)\right\rangle^{2}}=\int \mathrm{d} \boldsymbol{\mu} w(\boldsymbol{\mu}) \int \mathrm{d} \phi \mathrm{d} \phi^{\prime} P[\phi \mid \boldsymbol{\mu}] P\left[\phi^{\prime} \mid \boldsymbol{\mu}\right] \sin (\phi) \sin \left(\phi^{\prime}\right) .
\end{gathered}
$$

These four quantities are then compactified into the following two scalar observables ${ }^{12}$ :

$$
q=\frac{1}{2}\left(q_{c c}+q_{s s}\right), \quad m=\sqrt{m_{c}^{2}+m_{s}^{2}} .
$$

These two quantities are sufficient for characterizing any of the three anticipated phases $\{\mathrm{P}, \mathrm{F}$, $\mathrm{SG}\}$. In the absence of ferromagnetism our system is invariant under global rotations, which implies that $m_{c}=m_{s}=0$ and $q_{c c}=q_{s s}=q$. Furthermore, in the paramagnetic state we have $\left\langle\cos \left(\phi_{i}\right)\right\rangle=\left\langle\sin \left(\phi_{i}\right)\right\rangle=0$, so that we may write

$$
\mathrm{P}: \quad q=m=0 \quad \mathrm{~F}: \quad q>0, m>0 \quad \mathrm{SG}: \quad q>0, m=0 .
$$

The results of our numerical analyses are shown in figures 4,5 and 6 , and compared with simulation measurements. We find very satisfactory agreement between theory and

\footnotetext{
12 Many equivalent choices would have been possible. The present definitions have the advantage that they will generally give $\lim _{T \rightarrow 0} q=\frac{1}{2}$ and either $\lim _{T \rightarrow 0} m=0$ (in the SG state) or $\lim _{T \rightarrow 0} m=1$ (in the F state), so that we can always show both quantities together in one plot without loss of clarity.
} 

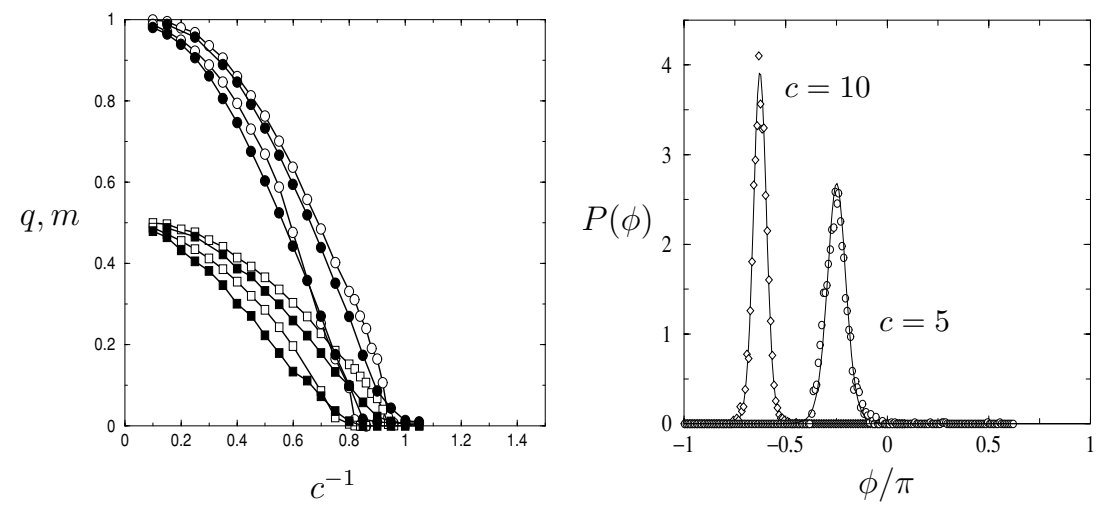

Figure 5. Population dynamics calculation of observables for strictly zero chiralities, namely $P(\omega)=\delta(\omega)$, and planar spins $(d=2)$. Left: the scalar observables $q=\frac{1}{2}\left(q_{c c}+q_{s s}\right)$ (connected open squares) and $m=\sqrt{m_{c}^{2}+m_{s}^{2}}$ (connected open circles) as functions of $c^{-1}$, and for $T / J=0.1,0.3$ (upper versus lower solid curve). Connected full circles and squares: simulation data, for $N=10^{5}$. Right: examples at $T / J=0.1$ of the observed distribution $P(\phi)=N^{-1} \sum_{i} \delta\left[\phi-\phi_{i}\right]$ in simulations (markers), together with the corresponding theoretical predictions (solid lines), for $c=10$ (left curves) and for $c=5$ (right curves). All observables were calculated with a population dynamics equation (52) and a truncated parametrization (see the main text for details).

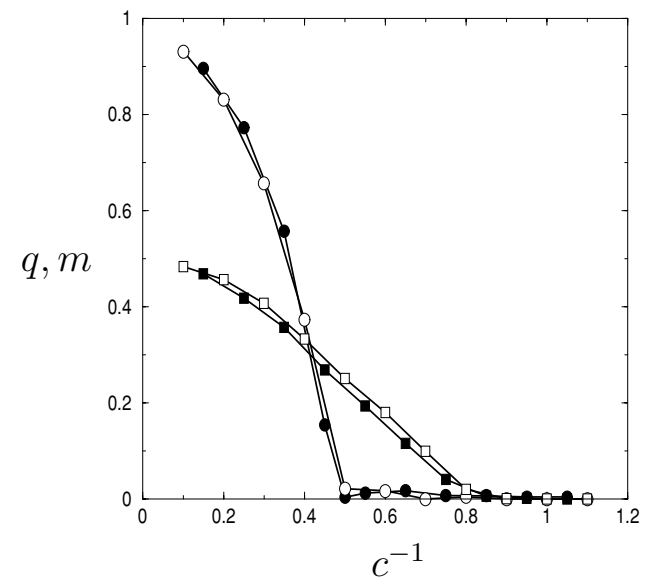

Figure 6. Population dynamics calculation of observables for binary distributed chiralities $P(\omega)=\frac{1}{2} \delta\left[\omega+\frac{\pi}{4}\right]+\frac{1}{2} \delta\left[\omega-\frac{\pi}{4}\right]$ and planar spins $(d=2): q=\frac{1}{2}\left(q_{c c}+q_{s s}\right)$ (connected open squares) and $m=\sqrt{m_{c}^{2}+m_{s}^{2}}$ (connected open circles) as functions of $c^{-1}$, and for $T / J=0.2$ (upper to lower solid curve). Full markers: simulation data (full squares: $q$; full circles: $m$ ), for $N=10^{5}$. The observables were calculated with a population dynamics equation (52) and a truncated parametrization (see the main text for details).

simulation experiments, in spite of the combined limitations imposed by the truncation of our parametrization in the population dynamics analysis, the inevitable equilibration difficulties of disordered spin systems near their transition points, and the finite system sizes in such simulations.

Figure 4 refers to the chirality distribution $P(\omega)=(2 \pi)^{-1}$, where we should find only the $\mathrm{P}$ and SG phases. This is borne out by the data: the order parameter $m$ is indeed consistently 
zero, and $q$ bifurcates to a non-zero value at more or less the predicted point. Also the locations of the transitions are as predicted by the corresponding phase diagram, i.e, figure 1 . In figure 5 we give data for $P(\omega)=\delta(\omega)$. Here all interactions are strictly ferromagnetic, leaving only the connectivity disorder, and the only possible phases are predicted to be $\mathrm{P}$ and $\mathrm{F}$. We do indeed observe the predicted non-zero magnetization for small values of $c^{-1}$, and again excellent agreement between population dynamics and simulations. In this figure, we also show the observed and predicted shapes ${ }^{13}$ of the spin angle distribution $P(\phi)=N^{-1} \sum_{i} \delta\left[\phi-\phi_{i}\right]$ (these are predicted by the theory to equal $P(\phi)=\int \mathrm{d} \boldsymbol{\mu} w(\boldsymbol{\mu}) P[\phi \mid \boldsymbol{\mu}]$ ), for two points in the ferromagnetic phase. In the SG phase such measurements tend to be more messy and prone to finite size effects, due to the inherent spread of the angles over the interval $[0,2 \pi]$. As expected, we see that an increase in the connectivity leads to a narrowing of the profile of $P(\phi)$, reflecting a stronger cooperative ordering of spin orientations. Once more the locations of the transitions are in agreement with the phase diagram, i.e., the left panel in figure 2. Finally, figure 6 corresponds to the binary chirality distribution $P(\omega)=\frac{1}{2} \delta\left[\omega+\frac{\pi}{4}\right]+\frac{1}{2} \delta\left[\omega-\frac{\pi}{4}\right]$, and temperature $T / J=0.2$. Here we may test our assumption regarding the location of the $\mathrm{F} \rightarrow$ $\mathrm{SG}$ transition line. The prediction of the phase diagram (the middle panel of figure 2 ) is to have phase $\mathrm{F}$ for $c^{-1}<\frac{1}{2}$, phase SG for $\frac{1}{2}<c^{-1}<0.798133$, and phase $\mathrm{P}$ for $c^{-1}>0.798133$. The agreement between population dynamics and simulations is once more very satisfactory: the magnetization $m$ and the spin-glass overlap $q$ indeed vanish more or less at the predicted $\mathrm{F} \rightarrow \mathrm{SG}$ and $\mathrm{SG} \rightarrow \mathrm{P}$ transition points.

The good agreement between the truncated population dynamics results and our numerical simulations underlines the correctness of (i) the RS order parameter equations themselves, (ii) the functional moment expansion used to locate the phase transitions $\mathrm{P} \rightarrow \mathrm{F}$ and $\mathrm{P} \rightarrow \mathrm{SG}$, and (iii) the assumed validity in the present models of the Parisi-Toulouse hypothesis regarding the location of the $\mathrm{F} \rightarrow \mathrm{SG}$ transition.

\section{General theory for $d>2$}

We next try to generalize the theoretical results obtained for $d=2$ to $d>2$. We will define the short-hand $\left|S_{d}\right|=\int_{S_{d}} \mathrm{~d} \sigma$. As expected, the paramagnetic solution of equation (16) for $\sigma \in S_{d-1}$ and $V(\sigma)=0$, is now seen to be $P(\sigma)=\left|S_{d-1}\right|^{-1}$ (as before, it is a solution for all $T$ ). Our analysis will involve generalizations of the modified Bessel functions, such as $I_{0, d}(z)=\left|S_{d-1}\right|^{-1} \int_{S_{d-1}} \mathrm{~d} \sigma \mathrm{e}^{z \sigma_{1}}$ (so that $\left.I_{0,2}(z)=I_{0}(z)\right)$.

\subsection{Guzai expansion for $d>2$}

To find bifurcations away from the paramagnetic state we put $P(\sigma) \rightarrow\left|S_{d-1}\right|^{-1}+\Delta(\sigma)$, with $W[\{P\}] \rightarrow \tilde{W}[\{\Delta\}]$ and $\tilde{W}[\{\Delta\}]=0$ as soon as $\int_{S_{d-1}} \mathrm{~d} \sigma \Delta(\sigma) \neq 0$ so that all probability densities are normalized, and we inspect the lowest order functional moments $\int\{\mathrm{d} \Delta\} \tilde{W}[\{\Delta\}] \Delta(\sigma)$ and $\int\{\mathrm{d} \Delta\} \tilde{W}[\{\Delta\}] \Delta\left(\sigma^{1}\right) \Delta\left(\sigma^{2}\right)$. Close to a continuous transition we assume there to be a small parameter $\epsilon$ such that $\int\{\mathrm{d} \Delta\} \tilde{W}[\{\Delta\}] \Delta\left(\sigma^{1}\right) \ldots \Delta\left(\sigma^{r}\right)=\mathcal{O}\left(\epsilon^{r}\right)$, as before. We note that

$$
\prod_{k=1}^{\ell} \int_{S_{d-1}} \mathrm{~d} \boldsymbol{\sigma}^{\prime}\left[\frac{1}{\left|S_{d-1}\right|}+\Delta_{k}\left(\boldsymbol{\sigma}^{\prime}\right)\right] \mathrm{e}^{\beta J \boldsymbol{\sigma} \cdot \mathbf{U}_{k} \boldsymbol{\sigma}^{\prime}}=\left[I_{0, d}(\beta J)\right]^{\ell} \prod_{k=1}^{\ell}\left[1+\frac{\int_{S_{d-1}} \mathrm{~d} \boldsymbol{\sigma}^{\prime} \Delta_{k}\left(\boldsymbol{\sigma}^{\prime}\right) \mathrm{e}^{\beta J \boldsymbol{\sigma} \cdot \mathbf{U}_{k} \boldsymbol{\sigma}^{\prime}}}{I_{0, d}(\beta J)}\right]
$$

\footnotetext{
13 Since the system is invariant under simultaneous rotations of all spins, the location of the maximum of the distribution $P(\phi)$ is completely free (only the shape carries information). To enable meaningful comparison, one therefore first has to position the theoretical curve such that its maximum coincides with that of the simulation data.
} 


$$
\begin{aligned}
= & {\left[I_{0, d}(\beta J)\right]^{\ell}\left[1+\sum_{k \leqslant \ell} \frac{\int_{S_{d-1}} \mathrm{~d} \boldsymbol{\sigma}^{\prime} \Delta_{k}\left(\boldsymbol{\sigma}^{\prime}\right) \mathrm{e}^{\beta J \sigma \cdot \mathbf{U}_{k} \boldsymbol{\sigma}^{\prime}}}{I_{0, d}(\beta J)}\right.} \\
& \left.+\frac{1}{2} \sum_{k \neq k^{\prime}}^{\ell} \frac{\int_{S_{d-1}} \mathrm{~d} \boldsymbol{\sigma}^{\prime} \Delta_{k}\left(\boldsymbol{\sigma}^{\prime}\right) \mathrm{e}^{\beta J \sigma \cdot \mathbf{U}_{k} \boldsymbol{\sigma}^{\prime}}}{I_{0, d}(\beta J)} \frac{\int_{S_{d-1}} \mathrm{~d} \boldsymbol{\sigma}^{\prime} \Delta_{k^{\prime}}\left(\boldsymbol{\sigma}^{\prime}\right) \mathrm{e}^{\beta J \sigma \cdot \mathbf{U}_{k^{\prime}} \boldsymbol{\sigma}^{\prime}}}{I_{0, d}(\beta J)}+\mathcal{O}\left(\epsilon^{3}\right)\right] .
\end{aligned}
$$

Integration of this expression over $\sigma \in S_{d-1}$ would eliminate the $\mathcal{O}(\epsilon)$ term, due to the constraint $\int_{S_{d-1}} \mathrm{~d} \sigma \Delta(\sigma)=0$. We may now expand the right-hand side of (16), and find

$$
\begin{aligned}
& \tilde{W}[\{\Delta\}]=\sum_{\ell \geqslant 0} \frac{c^{\ell}}{\ell !} \mathrm{e}^{-c} \int \prod_{k \leqslant \ell}\left[\left\{\mathrm{d} \Delta_{k}\right\} \tilde{W}\left[\left\{\Delta_{k}\right\}\right] \mathrm{d} \mathbf{U}_{k} P\left(\mathbf{U}_{k}\right)\right] \\
& \times \prod_{\sigma \in S_{d-1}} \delta\left[\Delta(\boldsymbol{\sigma})+\mathcal{O}\left(\epsilon^{3}\right)-\frac{1}{\left|S_{d-1}\right|} \sum_{k \leqslant \ell} \frac{\int_{S_{d-1}} \mathrm{~d} \boldsymbol{\sigma}^{\prime} \Delta_{k}\left(\boldsymbol{\sigma}^{\prime}\right) \mathrm{e}^{\beta J \sigma \cdot \mathbf{U}_{k} \boldsymbol{\sigma}^{\prime}}}{I_{0, d}(\beta J)}\right. \\
& +\frac{1}{2\left|S_{d-1}\right|} \sum_{k \neq k^{\prime}}^{\ell}\left\{\frac{\int_{S_{d-1}} \mathrm{~d} \boldsymbol{\sigma}^{\prime} \Delta_{k}\left(\boldsymbol{\sigma}^{\prime}\right) \mathrm{e}^{\beta J \sigma \cdot \mathbf{U}_{k} \boldsymbol{\sigma}^{\prime}}}{I_{0, d}(\beta J)} \frac{\int_{S_{d-1}} \mathrm{~d} \boldsymbol{\sigma}^{\prime} \Delta_{k^{\prime}}\left(\boldsymbol{\sigma}^{\prime}\right) \mathrm{e}^{\beta J \sigma \cdot \mathbf{U}_{k^{\prime}} \boldsymbol{\sigma}^{\prime}}}{I_{0, d}(\beta J)}\right. \\
& \left.\left.-\int \frac{d \boldsymbol{\sigma}^{\prime \prime}}{\left|S_{d-1}\right|} \frac{\int_{S_{d-1}} \mathrm{~d} \boldsymbol{\sigma}^{\prime} \Delta_{k}\left(\boldsymbol{\sigma}^{\prime}\right) \mathrm{e}^{\beta J \sigma^{\prime \prime} \cdot \mathbf{U}_{k} \boldsymbol{\sigma}^{\prime}}}{I_{0, d}(\beta J)} \frac{\int_{S_{d-1}} \mathrm{~d} \boldsymbol{\sigma}^{\prime} \Delta_{k^{\prime}}\left(\boldsymbol{\sigma}^{\prime}\right) \mathrm{e}^{\beta J \sigma^{\prime \prime} \cdot \mathbf{U}_{k^{\prime}} \boldsymbol{\sigma}^{\prime}}}{I_{0, d}(\beta J)}\right\}\right] .
\end{aligned}
$$

From this follow the relevant functional moment identities. We define $\Psi(\sigma)=$ $\int\{\mathrm{d} \Delta\} \tilde{W}[\{\Delta\}] \Delta(\boldsymbol{\sigma})$ and $\Psi\left(\boldsymbol{\sigma}^{1}, \boldsymbol{\sigma}^{2}\right)=\int\{\mathrm{d} \Delta\} \tilde{W}[\{\Delta\}] \Delta\left(\boldsymbol{\sigma}^{1}\right) \Delta\left(\boldsymbol{\sigma}^{2}\right)$. In lowest order $\epsilon$ one now finds the condition for a continuous $\mathrm{P} \rightarrow \mathrm{F}$ transition by solving the (constrained) eigenvalue problem

$$
\begin{aligned}
& \Psi(\boldsymbol{\sigma})=\frac{c}{I_{0, d}(\beta J)} \int_{S_{d-1}} \frac{\mathrm{d} \boldsymbol{\sigma}^{\prime}}{\left|S_{d-1}\right|} \Psi\left(\boldsymbol{\sigma}^{\prime}\right) \int \mathrm{d} \mathbf{U} P(\mathbf{U}) \mathrm{e}^{\beta J \sigma \cdot \mathbf{U} \sigma^{\prime}} \\
& \int_{S_{d-1}} \mathrm{~d} \boldsymbol{\sigma} \Psi(\boldsymbol{\sigma})=0 .
\end{aligned}
$$

If, on the other hand, the first order to bifurcate is $\epsilon^{2}$, we find a $\mathrm{P} \rightarrow \mathrm{SG}$ transition, marked by non-trivial solutions of the (constrained) eigenvalue problem

$$
\begin{aligned}
& \Psi\left(\sigma^{1}, \sigma^{2}\right)=\frac{c}{I_{0, d}^{2}(\beta J)} \int_{S_{d-1}} \frac{\mathrm{d} \tau^{1} \mathrm{~d} \tau^{2}}{\left|S_{d-1}\right|^{2}} \Psi\left(\boldsymbol{\tau}^{1}, \boldsymbol{\tau}^{2}\right) \int \mathrm{d} \mathbf{U} P(\mathbf{U}) \exp \left(\beta J\left(\boldsymbol{\sigma}^{1} \cdot \mathbf{U} \boldsymbol{\tau}^{1}+\boldsymbol{\sigma}^{2} \cdot \mathbf{U} \boldsymbol{\tau}^{2}\right)\right) \\
& \int_{S_{d-1}} \mathrm{~d} \boldsymbol{\sigma}^{1} \Psi\left(\boldsymbol{\sigma}^{1}, \boldsymbol{\sigma}^{2}\right)=\int_{S_{d-1}} \mathrm{~d} \boldsymbol{\sigma}^{2} \Psi\left(\sigma^{1}, \boldsymbol{\sigma}^{2}\right)=0
\end{aligned}
$$

For $d=2$ these expressions (60)-(63) reduce to those calculated earlier for $X Y$ spins, as they should. 


\subsection{Analysis of the bifurcation conditions}

First we turn to the $\mathrm{P} \rightarrow \mathrm{F}$ transition (60), (61). It will turn out advantageous to define two commuting linear operators $K$ and $L$ as follows:

$$
\begin{aligned}
& (K \Psi)(\boldsymbol{\sigma})=\int_{S_{d-1}} \frac{\mathrm{d} \boldsymbol{\tau}}{\left|S_{d-1}\right|} \mathrm{e}^{\beta J \sigma \cdot \tau} \Psi(\boldsymbol{\tau}) \\
& (L \Psi)(\boldsymbol{\sigma})=\int \mathrm{d} \mathbf{U} P(\mathbf{U}) \Psi\left(\mathbf{U}^{\dagger} \boldsymbol{\sigma}\right)
\end{aligned}
$$

These definitions allow us to write the eigenvalue problem (60), (61) as $K L \Psi=$ $c^{-1} I_{0, d}(\beta J) \Psi$, with constraint $\int_{S_{d-1}} \mathrm{~d} \sigma \Psi(\sigma)=0$. We can rewrite the action of $L$ as $(L \Psi)(\boldsymbol{\sigma})=\int \mathrm{d} \boldsymbol{\sigma}^{\prime} L\left(\boldsymbol{\sigma}, \boldsymbol{\sigma}^{\prime}\right) \Psi\left(\boldsymbol{\sigma}^{\prime}\right)$ with $L\left(\boldsymbol{\sigma}, \boldsymbol{\sigma}^{\prime}\right)=\int \mathrm{d} \mathbf{U} P(\mathbf{U}) \delta\left[\boldsymbol{\sigma}^{\prime}-\mathbf{U}^{\dagger} \boldsymbol{\sigma}\right]=$ $\int \mathrm{d} \mathbf{U} P(\mathbf{U}) \delta\left[\boldsymbol{\sigma}-\mathbf{U} \boldsymbol{\sigma}^{\prime}\right]$. The kernel $L\left(\boldsymbol{\sigma}, \boldsymbol{\sigma}^{\prime}\right)$ then represents the probability that a point $\boldsymbol{\sigma}^{\prime} \in S_{d-1}$ will be mapped onto $\sigma \in S_{d-1}$ by an orthogonal matrix from the ensemble $P(\mathbf{U})$. Both operators $K$ and $L$ are symmetric, as a consequence of $P(\mathbf{U})=P\left(\mathbf{U}^{\dagger}\right)$. Since $K$ and $L$ commute we may restrict ourselves to finding simultaneous eigenfunctions of $K$ and $L$.

It turns out that a similar strategy can be followed for the $\mathrm{P} \rightarrow \mathrm{SG}$ transition (62), (63). Here we have to define the commuting linear operators $K$ and $L$ as

$$
\begin{aligned}
& (K \Psi)\left(\boldsymbol{\sigma}^{1}, \boldsymbol{\sigma}^{2}\right)=\int_{S_{d-1}} \frac{\mathrm{d} \tau^{1} \mathrm{~d} \tau^{2}}{\left|S_{d-1}\right|^{2}} \exp \left(\beta J\left(\boldsymbol{\sigma}^{1} \cdot \tau^{1}+\boldsymbol{\sigma}^{2} \cdot \tau^{2}\right)\right) \Psi\left(\boldsymbol{\tau}^{1}, \boldsymbol{\tau}^{2}\right) \\
& (L \Psi)\left(\boldsymbol{\sigma}^{1}, \boldsymbol{\sigma}^{2}\right)=\int \mathrm{d} \mathbf{U} P(\mathbf{U}) \Psi\left(\mathbf{U}^{\dagger} \boldsymbol{\sigma}^{1}, \mathbf{U}^{\dagger} \boldsymbol{\sigma}^{2}\right)
\end{aligned}
$$

The eigenvalue problem (62), (63) can now be written as $K L \Psi=c^{-1} I_{0, d}^{2}(\beta J) \Psi$, with constraints $\int_{S_{d-1}} \mathrm{~d} \sigma^{1} \Psi\left(\sigma^{1}, \sigma^{2}\right)=\int_{S_{d-1}} \mathrm{~d} \sigma^{2} \Psi\left(\sigma^{1}, \sigma^{2}\right)=0$. Here we can rewrite the action of $L$ as $(L \Psi)\left(\sigma^{1}, \sigma^{2}\right)=\int \mathrm{d} \tau^{1} \mathrm{~d} \tau^{2} L\left(\sigma^{1}, \sigma^{2}, \tau^{1}, \tau^{2}\right) \Psi\left(\tau^{1}, \tau^{2}\right)$ with $L\left(\sigma^{1}, \sigma^{2}, \tau^{1}, \tau^{2}\right)=$ $\int \mathrm{d} \mathbf{U} P(\mathbf{U}) \delta\left[\boldsymbol{\sigma}^{1}-\mathbf{U} \tau^{1}\right] \delta\left[\boldsymbol{\sigma}^{2}-\mathbf{U} \tau^{2}\right]$. The kernel $L\left(\boldsymbol{\sigma}^{1}, \boldsymbol{\sigma}^{2}, \boldsymbol{\tau}^{1}, \boldsymbol{\tau}^{2}\right)$ now represents the probability that a pair of points $\left(\tau^{1}, \tau^{2}\right) \in S_{d-1} \otimes S_{d-1}$ will be mapped onto the pair $\left(\sigma^{1}, \sigma^{2}\right) \in S_{d-1} \otimes S_{d-1}$ by an orthogonal matrix from the ensemble $P(\mathbf{U})$. Both $K$ and $L$ are symmetric, as a consequence of $P(\mathbf{U})=P\left(\mathbf{U}^{\dagger}\right)$. Since they commute, we may once more restrict ourselves to finding simultaneous eigenfunctions of $K$ and $L$ individually.

At this stage it would appear appropriate to make a specific choice for the ensemble $P(\mathbf{U})$, for which we seek a controlled interpolation between having ferromagnetic and completely random chiral interactions. We may define this choice in terms of the above probability density $L\left(\sigma^{1}, \sigma^{2}, \tau^{1}, \tau^{2}\right)$ (from which the earlier kernel $L\left(\sigma, \sigma^{\prime}\right)$ follows by integration), for which we now choose the linear combination

$$
\begin{aligned}
& L\left(\boldsymbol{\sigma}^{1}, \boldsymbol{\sigma}^{2}, \boldsymbol{\tau}^{1},\right.\left.\boldsymbol{\tau}^{2}\right)=\epsilon \delta\left[\boldsymbol{\sigma}^{1}-\boldsymbol{\tau}^{1}\right] \delta\left[\boldsymbol{\sigma}^{2}-\boldsymbol{\tau}^{2}\right] \\
&+(1-\epsilon) \frac{\delta\left[\left|\boldsymbol{\sigma}^{1}\right|-1\right] \delta\left[\left|\boldsymbol{\sigma}^{2}\right|-1\right] \delta\left[\boldsymbol{\sigma}^{1} \cdot \boldsymbol{\sigma}^{2}-\boldsymbol{\tau}^{1} \cdot \boldsymbol{\tau}^{2}\right]}{\int_{S_{d-1}} \mathrm{~d} \boldsymbol{x} \mathrm{d} \mathbf{y} \delta[|\boldsymbol{x}|-1] \delta[|\mathbf{y}|-1] \delta\left[\boldsymbol{x} \cdot \mathbf{y}-\boldsymbol{\tau}^{1} \cdot \boldsymbol{\tau}^{2}\right]}
\end{aligned}
$$

In the non-ferromagnetic part of this measure, i.e., in the term proportional to $(1-\epsilon)$, we assign a uniform probability density to all combined image pairs $\left\{\sigma^{1}, \sigma^{2}\right\}$ of the vectors $\left\{\tau^{1}, \tau^{2}\right\}$ which preserve the inner products under the action of the random orthogonal matrices U. From definition (68) it then follows automatically upon integration over $\sigma^{2}$ that

$$
L(\boldsymbol{\sigma}, \boldsymbol{\tau})=\epsilon \delta[\boldsymbol{\sigma}-\boldsymbol{\tau}]+(1-\epsilon) \frac{\delta[|\boldsymbol{\sigma}|-1]}{\int_{S_{d-1}} \mathrm{~d} \boldsymbol{x} \delta[|\boldsymbol{x}|-1]} .
$$


For $\epsilon=1$ we return to a strictly ferromagnetic system; for $\epsilon=0$ we have fully and homogeneously distributed random chiral interactions.

The advantage of our choice (68) is that it allows us to diagonalize both kernels (66), (67) analytically. Working out the eigenvalue problem $\int_{S_{d-1}} \mathrm{~d} \sigma^{\prime} L\left(\sigma, \sigma^{\prime}\right) \Psi\left(\sigma^{\prime}\right)=\lambda \Psi(\sigma)$ shows that there are only two simple eigenspaces:

$\int_{S_{d-1}} \mathrm{~d} \sigma \Psi(\sigma) \neq 0: \quad \lambda=1 \quad$ and $\quad \int_{S_{d-1}} \mathrm{~d} \sigma \Psi(\sigma)=0: \quad \lambda=\epsilon$.

The first eigenspace is forbidden by constraint (61), so we may simply replace $L \rightarrow \epsilon \mathbb{1}$ in the eigenvalue problem for the $\mathrm{P} \rightarrow \mathrm{F}$ transition. Working out the $\mathrm{P} \rightarrow \mathrm{SG}$ eigenvalue problem $\int_{S_{d-1}} \mathrm{~d} \tau^{1} \mathrm{~d} \tau^{2} L\left(\sigma^{1}, \sigma^{2}, \tau^{1}, \tau^{2}\right) \Psi\left(\tau^{1}, \tau^{2}\right)=\lambda \Psi\left(\sigma^{1}, \sigma^{2}\right)$ leads to the following eigenspaces:

$\int_{S_{d-1}} \mathrm{~d} \sigma^{1} \mathrm{~d} \sigma^{2} \delta\left[\sigma^{1} \cdot \sigma^{2}-u\right] \Psi\left(\sigma^{1}, \sigma^{2}\right)=0, \quad$ for all $\quad u \in[-1,1]: \quad \lambda=\epsilon$

$\Psi\left(\sigma^{1}, \sigma^{2}\right)=\psi\left(\sigma^{1} \cdot \sigma^{2}\right), \quad$ for all $\sigma^{1}, \sigma^{2} \in S_{d-1}: \quad \lambda=1$.

It is clear that the $\lambda=1$ eigenspace is perfectly compatible with the constraints (63), which would, for example, be satisfied by any anti-symmetric function $\psi(u)$ in (72). Having solved the eigenvalue problem for the operators $L$ for the choice of ensemble (68), we may concentrate on the following reduced eigenvalue problems from which to extract the phase transitions away from the paramagnetic state:

$$
\begin{aligned}
& \mathrm{P} \rightarrow \mathrm{F}: \quad \int_{S_{d-1}} \frac{\mathrm{d} \tau}{\left|S_{d-1}\right|} \mathrm{e}^{\beta J \sigma \cdot \tau} \Psi(\tau)=\frac{I_{0, d}(\beta J)}{\epsilon c} \Psi(\sigma) \\
& \text { constraint : } \quad \int_{S_{d-1}} \mathrm{~d} \sigma \Psi(\sigma)=0 \\
& \mathrm{P} \rightarrow \mathrm{SG}_{a}: \quad \int_{S_{d-1}} \frac{\mathrm{d} \tau^{1} \mathrm{~d} \tau^{2}}{\left|S_{d-1}\right|^{2}} \exp \left(\beta J\left(\sigma^{1} \cdot \tau^{1}+\sigma^{2} \cdot \tau^{2}\right)\right) \Psi\left(\tau^{1}, \tau^{2}\right)=\frac{I_{0, d}^{2}(\beta J)}{\epsilon c} \Psi\left(\sigma^{1}, \sigma^{2}\right) \\
& \int_{S_{d-1}} \mathrm{~d} \sigma^{1} \mathrm{~d} \sigma^{2} \delta\left[\sigma^{1} \cdot \sigma^{2}-u\right] \Psi\left(\sigma^{1}, \sigma^{2}\right)=0, \quad \text { for all } u \in[-1,1] \\
& \text { constraints : } \quad \int_{S_{d-1}} \mathrm{~d} \sigma^{1} \Psi\left(\sigma^{1}, \sigma^{2}\right)=\int_{S_{d-1}} \mathrm{~d} \sigma^{2} \Psi\left(\sigma^{1}, \sigma^{2}\right)=0 \\
& \mathrm{P} \rightarrow \mathrm{SG}_{b}: \quad \int_{S_{d-1}} \frac{\mathrm{d} \tau^{1} \mathrm{~d} \tau^{2}}{\left|S_{d-1}\right|^{2}} \exp \left(\beta J\left(\sigma^{1} \cdot \tau^{1}+\boldsymbol{\sigma}^{2} \cdot \tau^{2}\right)\right) \psi\left(\tau^{1} \cdot \tau^{2}\right)=\frac{I_{0, d}^{2}(\beta J)}{c} \psi\left(\sigma^{1} \cdot \sigma^{2}\right) \\
& \text { constraint : } \quad \int_{S_{d-1}} \mathrm{~d} \sigma \psi\left(\sigma_{1}\right)=0 \text {. }
\end{aligned}
$$

Although they cannot formally be ruled out, we will henceforth disregard the $\mathrm{P} \rightarrow \mathrm{SG}_{a}$ transitions, since they are less likely to correspond to the largest eigenvalue in view of the extra factor $\epsilon$ involved, and because in addition the associated constraints (infinite in number) would appear to severely limit the space of allowed functions ${ }^{14}$.

\footnotetext{
${ }^{14}$ There are further reasons to reduce the likelihood of the $\mathrm{P} \rightarrow \mathrm{SG}_{a}$ transition being physical. For instance, for $d=2$ the eigenfunctions can only depend on the inner product between the two vectors involved, hence here one simply cannot satisfy the constraints of the $\mathrm{P} \rightarrow \mathrm{SG}_{a}$ bifurcation.
} 


\subsection{Explicit results for $d=3$}

We finally work out our previous general equations for the value $d=3$, where we may turn to polar coordinates and write our integration variables as $\sigma=(\sin (\theta) \cos (\phi)$, $\sin (\theta) \sin (\phi), \cos (\theta))$ with $\phi \in[-\pi, \pi]$ and $\theta \in[0, \pi]$. We note that $I_{0,3}(x)=\sinh (x) / x$. Insertion of the polar coordinate representation in our general eigenvalue equations shows that for $d=3$ the second-order $\mathrm{P} \rightarrow \mathrm{F}$ transition is to be solved from

$$
\begin{gathered}
\int_{0}^{\pi} \frac{\mathrm{d} \theta^{\prime} \sin \left(\theta^{\prime}\right)}{2} \int_{-\pi}^{\pi} \frac{\mathrm{d} \phi^{\prime}}{2 \pi} \exp \left(\beta J\left[\sin (\theta) \sin \left(\theta^{\prime}\right) \cos \left(\phi^{\prime}\right)+\cos (\theta) \cos \left(\theta^{\prime}\right)\right]\right) \Psi\left(\theta^{\prime}, \phi+\phi^{\prime}\right) \\
=\frac{\sinh (\beta J)}{\epsilon \beta J c} \Psi(\theta, \phi) .
\end{gathered}
$$

We observe that the solutions of this equation are of the form $\Psi(\theta, \phi)=\rho(\cos (\theta)) \mathrm{e}^{\mathrm{i} k \phi}$ with $k \in \mathbb{N}$ (similar to, for example, the spherical harmonics $Y_{\ell, m}(\theta, \phi)$, but with, as we will find below, a different dependence on $\theta$ ), and with the function $\rho$ to be solved from

$$
\begin{aligned}
& \int_{-1}^{1} \frac{\mathrm{d} y}{2} I_{k}\left(\beta J \sqrt{1-x^{2}} \sqrt{1-y^{2}}\right) \mathrm{e}^{\beta J x y} \rho(y)=\frac{\sinh (\beta J)}{\epsilon \beta J c} \rho(x) \\
& \text { constraint : } \quad \text { either } k \neq 0 \text { or } \quad \int_{-1}^{1} \mathrm{~d} x \rho(x)=0 .
\end{aligned}
$$

The kernel in (77) of which we seek the eigenfunctions is invariant under parity transformation, so we may restrict ourselves to eigenfunctions $\rho(x)$ which are either symmetric, $\rho_{+}(x)$, or anti-symmetric, $\rho_{-}(x)$. Upon implementing these restrictions we find

$$
\begin{array}{ll}
\rho_{+}(x): & \int_{0}^{1} \mathrm{~d} y I_{k}\left(\beta J \sqrt{1-x^{2}} \sqrt{1-y^{2}}\right) \cosh (\beta J x y) \rho(y)=\frac{\sinh (\beta J)}{\epsilon \beta J c} \rho(x) \\
\rho_{-}(x): & \int_{0}^{1} \mathrm{~d} y I_{k}\left(\beta J \sqrt{1-x^{2}} \sqrt{1-y^{2}}\right) \sinh (\beta J x y) \rho(y)=\frac{\sinh (\beta J)}{\epsilon \beta J c} \rho(x) .
\end{array}
$$

It should be expected that, as for $d=2$, the physical (i.e., largest) eigenvalue is the one with the lowest allowed value of $k$, which here is $k=0$. Furthermore, we note that for $\rho_{-}(x)$ the constraint in (73) is automatically satisfied. We have not been able yet to solve the above eigenvalue problem analytically, and have instead simply resorted to numerical evaluation of the largest eigenvalue in (34). This shows that the largest eigenvalue is indeed found for $k=0$ and $\rho_{-}(x)$.

Our analysis of the $\mathrm{P} \rightarrow \mathrm{SG}_{b}$ transition can be simplified if we use the fact that (75) is written strictly in terms of inner products of the various unit-length vectors. This allows us to choose a convenient basis, for example, one where $\sigma^{2}=(0,0,1)$. Upon again using polar coordinates to do the integrations over the sphere $S_{2}$, we find that for $d=3$ the second-order $\mathrm{P} \rightarrow \mathrm{SG}$ transition is to be solved from

$$
\begin{aligned}
\int_{0}^{\pi} \frac{\mathrm{d} \theta \sin (\theta)}{2} & \int_{-\pi}^{\pi} \frac{\mathrm{d} \phi}{2 \pi} \int_{0}^{\pi} \frac{\mathrm{d} \theta^{\prime} \sin \left(\theta^{\prime}\right)}{2} \int_{-\pi}^{\pi} \frac{\mathrm{d} \phi^{\prime}}{2 \pi} \exp \left(\beta J \left[\cos (\theta)+x \cos \left(\theta^{\prime}\right)\right.\right. \\
& \left.\left.+\sin \left(\theta^{\prime}\right) \cos \left(\phi^{\prime}\right) \sqrt{1-x^{2}}\right]\right) \psi\left(\sin (\theta) \sin \left(\theta^{\prime}\right) \cos (\phi)+\cos (\theta) \cos \left(\theta^{\prime}\right)\right) \\
= & \frac{\sinh ^{2}(\beta J)}{c(\beta J)^{2}} \psi(x) .
\end{aligned}
$$



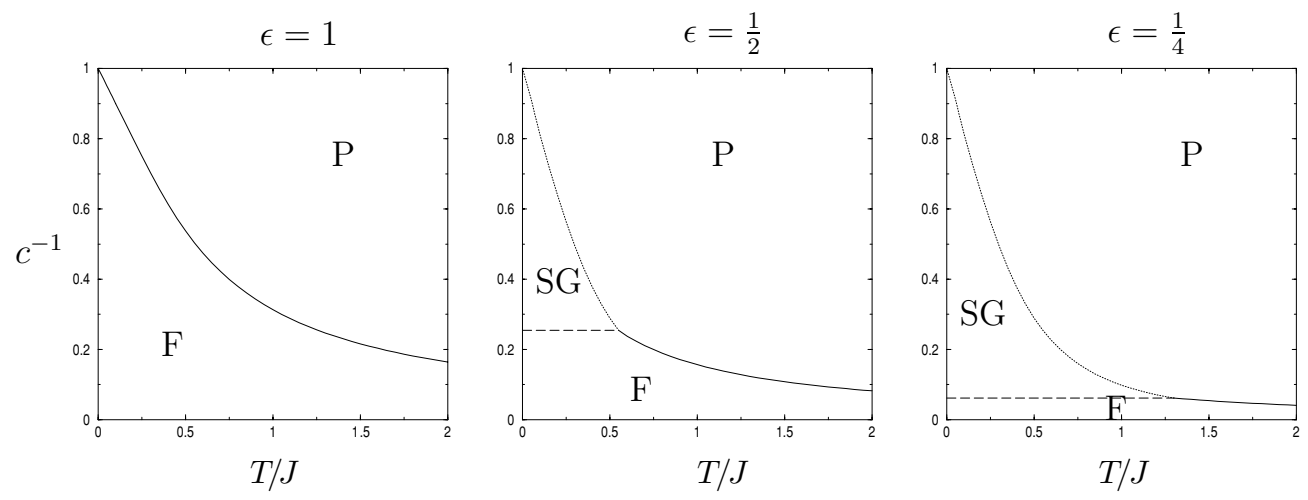

Figure 7. Phase diagrams in $d=3$, for the orthogonal random matrix ensemble characterized by equation (68), and for different values of $\epsilon . \epsilon=1$ corresponds to purely ferromagnetic interactions, whereas for $\epsilon=0$ they are fully random. As in the case $d=2$, except for the location of the triple point, the $\mathrm{SG} \rightarrow \mathrm{F}$ transitions cannot be obtained from our present functional moment expansions, but have been inferred from the assumption that there is no change of phase character after the onset of order, when the temperature is decreased further for fixed connectivity $c$.

Via suitable transformations of variables, namely, $t=\cos (\theta)$ and $s=\cos \left(\theta^{\prime}\right)$, and insertion of $\int \mathrm{d} y \delta\left[y-\sin (\theta) \sin \left(\theta^{\prime}\right) \cos (\phi)-\cos (\theta) \cos \left(\theta^{\prime}\right)\right]$ this expression can be simplified to

$$
\begin{aligned}
\int_{-1}^{1} \frac{\mathrm{d} y \mathrm{~d} s \mathrm{~d} t}{4 \pi} & I_{0}\left(\beta J \sqrt{1-s^{2}} \sqrt{1-x^{2}}\right) \mathrm{e}^{\beta J[s x+t]} \frac{\theta\left[\left(1-s^{2}\right)\left(1-t^{2}\right)-(y-s t)^{2}\right]}{\sqrt{\left(1-s^{2}\right)\left(1-t^{2}\right)-(y-s t)^{2}}} \psi(y) \\
= & \frac{\sinh ^{2}(\beta J)}{c(\beta J)^{2}} \psi(x) .
\end{aligned}
$$

This latter equation is to be solved subject to the constraint $\int_{-1}^{1} \mathrm{~d} y \psi(y)=0$. The integration kernel in (80) is again symmetric, yielding as before either strictly symmetric or strictly antisymmetric eigenfunctions. Again the anti-symmetric eigenfunctions offer the advantage of automatically satisfying the appropriate constraint.

We are now in a position to construct phase diagrams for $d=3$ and the orthogonal random matrix ensemble characterized by (68), by solving the remaining two eigenvalue problems (79), (80) with their associated constraints numerically. We have done this for three different values of $\epsilon$, leading to the phase diagrams in figure 7, which can be compared to the $d=2$ results of figure 2. As might be expected on physical grounds, the extra degrees of freedom available to each spin in $d=3$ (which increase the potential for the system to minimize its free energy entropically, as opposed to energetically) lead to a lower transition temperature to an ordered state, be it spin-glass or ferromagnetic.

\subsection{Numerical calculation of order parameters via population dynamics}

Let us now turn to the numerical evaluation of the order parameters in our system. In a spirit similar to that of the case $d=2$, i.e., section 5.2, we will extract the relevant population dynamics equations from (17) upon making a suitable choice for the parametrization of $P[\boldsymbol{\sigma} \mid \boldsymbol{\mu}]$. Here we will again use the family of Fourier modes 


$$
\begin{aligned}
P(\phi, \theta \mid \boldsymbol{\mu})= & \frac{1}{D(\boldsymbol{\mu})} \exp \left(\sum_{m \geqslant 1}\left[A_{m}^{c} \cos (m \phi)+A_{m}^{s} \sin (m \phi)+B_{m}^{c} \cos (m \theta)+B_{m}^{s} \sin (m \theta)\right]\right) \\
& \times \exp \left(\sum _ { m , m ^ { \prime } \geqslant 1 } \left[H_{m m^{\prime}}^{c c} \cos (m \phi) \cos \left(m^{\prime} \theta\right)+H_{m m^{\prime}}^{c s} \cos (m \phi) \sin \left(m^{\prime} \theta\right)\right.\right. \\
& \left.\left.+H_{m m^{\prime}}^{s c} \sin (m \phi) \cos \left(m^{\prime} \theta\right)+H_{m m^{\prime}}^{s s} \sin (m \phi) \sin \left(m^{\prime} \theta\right)\right]\right)
\end{aligned}
$$

where $D(\boldsymbol{\mu})$ is the relevant normalization constant and $\boldsymbol{\mu}$ denotes collectively all coefficients $\left\{A^{\star}, B^{\star}, H^{\star \star}\right\}$ with $\star \in\{c, s\}$. From this point, one can proceed further by working out expression (17) and converting it into one for the coefficients $\left\{A^{\star}, B^{\star}, H^{\star \star}\right\}$. We have implemented this strategy on the basis of a truncation after eight coefficients, i.e., we have taken $A_{m}^{\star}=A^{\star} \delta_{m, 1}, B_{m}^{\star}=B^{\star} \delta_{m, 1}$ and $H_{m m^{\prime}}^{\star \star}=H^{\star \star} \delta_{m, 1} \delta_{m^{\prime}, 1}$ so that $\boldsymbol{\mu}=\left(A^{c}, A^{s}, B^{c}, B^{s}\right.$, $H^{c c}, H^{c s}, H^{s c}, H^{s s}$ ) (details of the resulting expressions can be found in the appendix). The self-consistent equation for $w(\boldsymbol{\mu})$ that follows can then be solved using a population dynamics prescription.

Finally, given the stationary distribution of coefficients $w(\boldsymbol{\mu})$ in the population dynamics method and expression (81) one can then evaluate the order parameters of the system, e.g., the magnetization and the spin-glass order parameter

$$
m=\sqrt{m_{x}^{2}+m_{y}^{2}+m_{z}^{2}} \quad q=\frac{1}{3}\left(q_{x}+q_{y}+q_{z}\right)
$$

where now

$$
\begin{array}{ll}
m_{x}=\left\langle\langle\cos (\phi) \sin (\theta)\rangle_{\phi, \theta \mid \boldsymbol{\mu}}\right\rangle_{\mu} & q_{x}=\left\langle\langle\cos (\phi) \sin (\theta)\rangle_{\phi, \theta \mid \mu}^{2}\right\rangle_{\mu} \\
m_{y}=\left\langle\langle\sin (\phi) \sin (\theta)\rangle_{\phi, \theta \mid \boldsymbol{\mu}}\right\rangle_{\mu} \quad q_{y}=\left\langle\langle\sin (\phi) \sin (\theta)\rangle_{\phi, \theta \mid \boldsymbol{\mu}}^{2}\right\rangle_{\boldsymbol{\mu}} \\
m_{z}=\left\langle\langle\cos (\theta)\rangle_{\phi, \theta \mid \boldsymbol{\mu}}\right\rangle_{\mu} \quad q_{z}=\left\langle\langle\cos (\theta)\rangle_{\phi, \theta \mid \boldsymbol{\mu}}^{2}\right\rangle_{\boldsymbol{\mu}}
\end{array}
$$

and with the averages

$$
\begin{aligned}
& \langle(\cdots)\rangle_{\phi, \theta \mid \boldsymbol{\mu}}=\int_{-\pi}^{\pi} \mathrm{d} \phi \int_{0}^{\pi} \mathrm{d} \theta|\sin (\theta)| P(\phi, \theta \mid \boldsymbol{\mu})(\cdots) \\
& \langle(\cdots)\rangle_{\boldsymbol{\mu}}=\int \mathrm{d} \boldsymbol{\mu} w(\boldsymbol{\mu})(\cdots) .
\end{aligned}
$$

In figure 8 we show the results of evaluating our observables numerically via the above strategy. We compare our bifurcation analysis (leading to predictions for the locations of phase transitions) with the outcome of population dynamics analysis and with data measured in simulation experiments. In practice, our population dynamics shows good convergence already for relatively modest population sizes of 2000 fields.

The Monte Carlo simulations were done in the present case $d=3$ using the heat-bath algorithm of $[44,45]$, and with system sizes of $10^{5}$ spins. To generate an ensemble of uniformly distributed random orthogonal matrices it is convenient to represent the rotation matrices using Euler angles $(\alpha, \beta, \gamma)$ (in standard notation), i.e., $\mathbf{U}=R_{z}(\alpha) R_{y}(\beta) R_{z}(\gamma)$ for any $\mathbf{U} \in S O(3)$. Then, uniform integration over the Lie group $S O(3)$ is given by the Haar measure $\mathrm{d}_{H}(\alpha, \beta, \gamma)$ which, in Euler representation, takes the form $\mathrm{d} g_{H}(\alpha, \beta, \gamma)=\left(8 \pi^{2}\right)^{-1} \mathrm{~d} \alpha \mathrm{d} \beta \mathrm{d} \gamma \sin (\beta)$, with $\alpha, \gamma \in[0,2 \pi]$ and $\beta \in[0, \pi]$. The relevant matrix distribution $P(\mathbf{U})$ can then be written as 

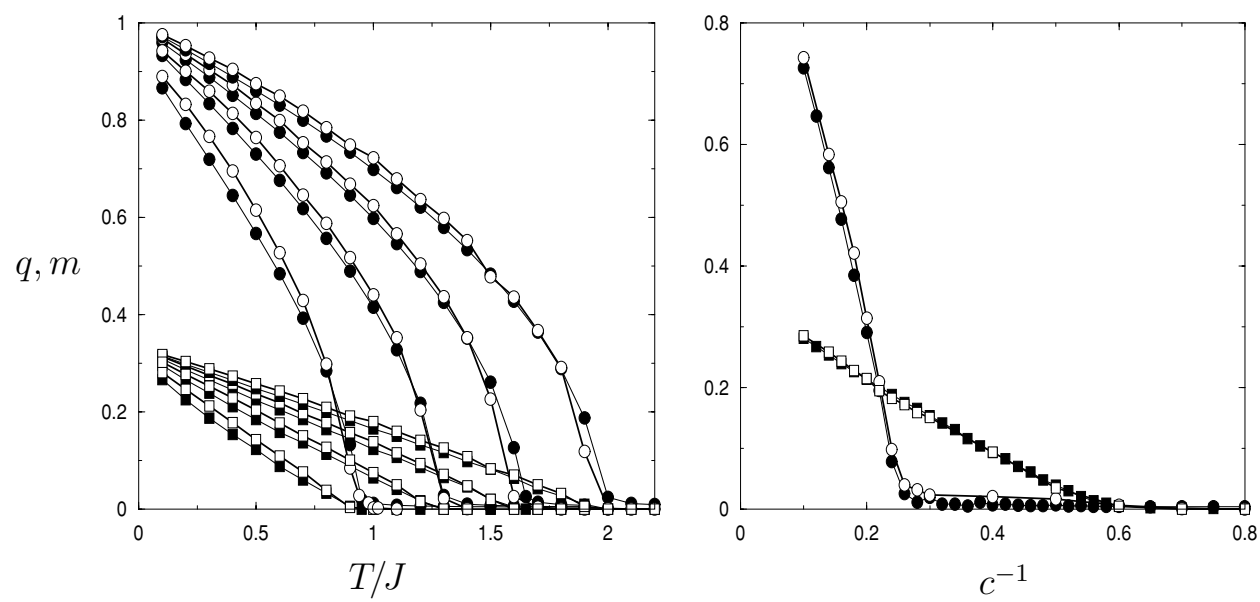

Figure 8. Comparison between theoretical predictions, population dynamics (open symbols) and Monte Carlo simulations (solid symbols) for $d=3$. Left picture: we show the magnetization $m$ (circles) and the spin-glass order parameter $q$ (squares) defined in (82), for $\epsilon=1$ and for connectivity values $c=3,4,5,6$ (from left to right). The agreement indicates that our truncation in the parametrization of (81) does not have a significant impact on the numerical accuracy. Right picture: the order parameters $m$ (circles) and $q$ (squares) for $\epsilon=1 / 2$, along the line $T / J=0.25$. The magnetization is seen to become zero around $c^{-1}=0.25$, thus verifying our assumption for the location of the SG-F line. All simulations were done for $N=10^{5}$ spins.

$P(\mathbf{U})=\epsilon \delta[\mathbf{U}-\mathbb{1}]+\frac{1-\epsilon}{8 \pi^{2}} \int_{0}^{2 \pi} \mathrm{d} \alpha \int_{0}^{\pi} \mathrm{d} \beta \int_{0}^{2 \pi} \mathrm{d} \gamma \sin (\beta) \delta\left[\mathbf{U}-R_{z}(\alpha) R_{y}(\beta) R_{z}(\gamma)\right]$

with

$$
\begin{aligned}
& R_{z}(\alpha)=\left(\begin{array}{ccc}
\cos \alpha & \sin \alpha & 0 \\
-\sin \alpha & \cos \alpha & 0 \\
0 & 0 & 1
\end{array}\right) \\
& R_{z}(\gamma)=\left(\begin{array}{ccc}
\cos \gamma & \sin \gamma & 0 \\
-\sin \gamma & \cos \gamma & 0 \\
0 & 0 & 1
\end{array}\right) .
\end{aligned}
$$

In the left picture of figure 8 we show the magnetization and spin-glass order parameters (82) for the case where $\epsilon=1$, i.e., where our rotation matrices reduce to the unit matrix $\mathbf{U}=\mathbb{1}$ and the only source of disorder in the system is the random nature of the connectivity variables $\left\{c_{i j}\right\}$. Here both the population dynamics and the simulation experiments reproduce the $\mathrm{P} \rightarrow \mathrm{F}$ transition at the predicted connectivity value. The excellent agreement obtained indicates that, in retrospect, our truncation of the parametrization (81) has been made with only a minor cost in accuracy. In the more involved scenarios where $\epsilon<1$, a spin-glass phase with $m=0$ and $q>0$ will become possible. An example is shown in the right picture of figure 8 , where we chose the values $\epsilon=1 / 2$ and $T / J=0.25$. For these parameter values our theory predicts a P-SG transition, but also, based on physical grounds (i.e., the absence of re-entrance phenomena) we have assumed that the elusive F-SG transition is located at the line segment parallel to the $T$-axis, connecting the triple point where all phases meet to the point $T / J=0$. In figure 8 we see that also this latter assumption is verified numerically (by the population dynamics results), and that the simulation data are, in turn, again in good agreement with those of the population dynamics. 


\section{Discussion}

In this paper, we have applied the equilibrium replica method as developed for finitely connected scalar spin systems to models of finitely connected unit-length vectorial spins of dimension $d$, with pair-interactions which are given by random orthogonal $d \times d$ matrices. Since our spins are continuous and the connectivity $c$ is finite, rather than an effective field distribution (as would have been the case for finitely connected discrete scalar spins), here the replica-symmetric order parameter is a functional. This generates a number of technical complications. Firstly, rather than finding continuous transitions away from the paramagnetic state by expansion of the replica-symmetric (RS) order parameter in powers of (scalar) moments of a field distribution, here we have to generalize this procedure to an expansion in functional moments. Secondly, one should expect serious numerical difficulties when attempting to solve numerically the RS order parameter functional from the appropriate self-consistent nonlinear population dynamics equation. Here, however, these difficulties are kept under control due to the constrained nature of the microscopic degrees of freedom. Since the present continuous spins live on the sphere $S_{d-1}$, their value domain is bounded, and it is therefore possible to construct efficient and relatively accurate low-dimensional parametrizations of the order parameter functional (in contrast to the case of unbounded value domains, as, for example, with ordinary soft spins).

We have developed our theory initially for arbitrary values of the dimension $d$ of the spins, and arbitrary choices of the ensemble of random orthogonal matrices. However, we ultimately calculate phase diagrams and the values of moments of the order parameter explicitly for $d=2$ (where our models reduce to finitely connected $X Y$ spins with random chiral interactions) and for $d=3$ (where they reduce to finitely connected classical Heisenberg spins with random chiral interactions). For $d=2,3$ we find three types of phases: a paramagnetic $(\mathrm{P})$, a ferromagnetic $(\mathrm{F})$, and a spin-glass phase (SG). The calculation of all continuous $\mathrm{P} \rightarrow \mathrm{F}$ and $\mathrm{P} \rightarrow \mathrm{SG}$ transition lines can in all cases be reduced to the (numerical) solution of relatively simple functional eigenvalue problems; the $\mathrm{F} \rightarrow \mathrm{SG}$ transition is constructed from the location of the triple point, in combination with the conjecture (based on previous experience with similar systems, and in line with the Parisi-Toulouse hypothesis [41] for the RSB solution) that the phase entered at the onset of order (upon leaving the paramagnetic state) will continue to hold upon lowering the temperature further for fixed connectivity $c$. The calculation of observables in the F and SG phases was carried out using population dynamics techniques, applied to (truncated) parametrizations of the order parameter functional, and the results were tested against numerical simulations to reveal excellent agreement.

It should be emphasized that for bond-disordered spin systems such as those studied here one should not expect a replica-symmetric theory to be correct at low temperatures. The fact that in comparing the predictions of our RS calculations with numerical simulations this has not yet been apparent suggests that RSB effects at the level of order parameters such as $\left\{m_{c}, m_{s}, q_{c c}, q_{s s}\right\}$ are confined to temperatures $T / J<0.1$. In addition, one would have expected to see RSB effects in the location of the F $\rightarrow$ SG transition line; however, even within the RS ansatz the latter cannot yet be calculated with the mathematical tools presently available (which is why we turned to the RSB-based Parisi-Toulouse hypothesis).

We believe the main deliverables of this study to be the successful extension and application of finite connectivity replica techniques to more demanding scenarios, where the microscopic equilibrated degrees of freedom are neither discrete nor of a scalar nature, and where also their interactions are of a mathematically more complicated form than just weighted (inner) products. These techniques, which could easily be adapted to non-Poissonnian random 
connectivity graphs, are not only useful tools in the study of physical systems, but may also be helpful to analytically determine, for example, the influence of topology on global processes in non-physical systems with scale-free connectivity, such as the synchronization of randomly and finitely connected planar oscillators [43].

\section{Acknowledgments}

This study was initiated during an informal Finite Connectivity Workshop at King's College London in November 2003. The authors acknowledge financial support from the the ESF SPHINX programme, the Ministerio de Educación, Cultura y Deporte (Spain, grant no SB2002-0107), the Engineering and Physical Sciences Research Council, the MCYT (Spain, grant no BFM2003-08258), the FOM Foundation (Fundamenteel Onderzoek der Materie, the Netherlands), and the State Scholarships Foundation (Greece).

\section{Appendix. Details of population dynamics for $d=3$}

To arrive at a numerically tractable form of the population dynamics relations, one is required to truncate (81) to a relatively small number of coefficients. Here we took

$$
\begin{aligned}
P(\phi, \theta \mid \boldsymbol{\mu})= & \frac{1}{D(\boldsymbol{\mu})} \exp \left[A^{c} \cos (\phi)+A^{s} \sin (\phi)+B^{c} \cos (\theta)+B^{s} \sin (\theta)\right] \\
& \times \exp \left[H^{c c} \cos (\phi) \cos (\theta)+H^{c s} \cos (\phi) \sin (\theta)\right. \\
& \left.+H^{s c} \sin (\phi) \cos (\theta)+H^{s s} \sin (\phi) \sin (\theta)\right]
\end{aligned}
$$

with $D(\boldsymbol{\mu})$ the appropriate normalization constant. The update relations for the above coefficients follow from the orthogonality relations of the trigonometric functions,

$$
\begin{aligned}
w(\boldsymbol{\mu})= & \sum_{\ell \geqslant 0} \frac{\mathrm{e}^{-c} c^{\ell}}{\ell !} \int\left[\prod_{k \leqslant \ell} \mathrm{d} \boldsymbol{\mu}_{k} w\left(\boldsymbol{\mu}_{k}\right) \mathrm{d} \mathbf{U}_{k} P\left(\mathbf{U}_{k}\right)\right] \\
& \times \delta\left[A^{c}-\sum_{k \leqslant \ell} \frac{\pi K_{c}\left(\boldsymbol{\mu}_{k}, \mathbf{U}_{k}\right)-4 M_{c s}\left(\boldsymbol{\mu}_{k}, \mathbf{U}_{k}\right)}{\pi\left(\pi^{2}-8\right)}\right] \\
& \times \delta\left[A^{s}-\sum_{k \leqslant \ell} \frac{\pi K_{s}\left(\boldsymbol{\mu}_{k}, \mathbf{U}_{k}\right)-4 M_{s s}\left(\boldsymbol{\mu}_{k}, \mathbf{U}_{k}\right)}{\pi\left(\pi^{2}-8\right)}\right] \\
& \times \delta\left[B^{c}-\sum_{k \leqslant \ell} \frac{1}{\pi^{2}} V_{c}\left(\boldsymbol{\mu}_{k}, \mathbf{U}_{k}\right)\right] \delta\left[B^{s}-\sum_{k \leqslant \ell} \frac{\pi V_{s}\left(\boldsymbol{\mu}_{k}, \mathbf{U}_{k}\right)-2 M\left(\boldsymbol{\mu}_{k}, \mathbf{U}_{k}\right)}{\pi\left(\pi^{2}-8\right)}\right] \\
& \times \delta\left[H^{c c}-\sum_{k \leqslant \ell} \frac{2}{\pi^{2}} M_{c c}\left(\boldsymbol{\mu}_{k}, \mathbf{U}_{k}\right)\right] \delta\left[H^{c s}-\sum_{k \leqslant \ell} 2 \frac{\pi M_{c s}\left(\boldsymbol{\mu}_{k}, \mathbf{U}_{k}\right)-2 K_{c}\left(\boldsymbol{\mu}_{k}, \mathbf{U}_{k}\right)}{\pi\left(\pi^{2}-8\right)}\right] \\
& \times \delta\left[H^{s c}-\sum_{k \leqslant \ell} \frac{2}{\pi^{2}} M_{s c}\left(\boldsymbol{\mu}_{k}, \mathbf{U}_{k}\right)\right] \delta\left[H^{s s}-\sum_{k \leqslant \ell} 2 \frac{\pi M_{s s}\left(\boldsymbol{\mu}_{k}, \mathbf{U}_{k}\right)-2 K_{s}\left(\boldsymbol{\mu}_{k}, \mathbf{U}_{k}\right)}{\pi\left(\pi^{2}-8\right)}\right]
\end{aligned}
$$

with the abbreviations

$$
K_{c}\left(\boldsymbol{\mu}_{k}, \mathbf{U}_{k}\right)=\int_{-\pi}^{\pi} \mathrm{d} \phi \cos (\phi) \int_{0}^{\pi} \mathrm{d} \theta R\left(\phi, \theta ; \boldsymbol{\mu}_{k}, \mathbf{U}_{k}\right)
$$




$$
\begin{aligned}
& K_{s}\left(\boldsymbol{\mu}_{k}, \mathbf{U}_{k}\right)=\int_{-\pi}^{\pi} \mathrm{d} \phi \sin (\phi) \int_{0}^{\pi} \mathrm{d} \theta R\left(\phi, \theta ; \boldsymbol{\mu}_{k}, \mathbf{U}_{k}\right) \\
& V_{c}\left(\boldsymbol{\mu}_{k}, \mathbf{U}_{k}\right)=\int_{-\pi}^{\pi} \mathrm{d} \phi \int_{0}^{\pi} \mathrm{d} \theta \cos (\theta) R\left(\phi, \theta ; \boldsymbol{\mu}_{k}, \mathbf{U}_{k}\right) \\
& V_{s}\left(\boldsymbol{\mu}_{k}, \mathbf{U}_{k}\right)=\int_{-\pi}^{\pi} \mathrm{d} \phi \int_{0}^{\pi} \mathrm{d} \theta \sin (\theta) R\left(\phi, \theta ; \boldsymbol{\mu}_{k}, \mathbf{U}_{k}\right) \\
& M_{c c}\left(\boldsymbol{\mu}_{k}, \mathbf{U}_{k}\right)=\int_{-\pi}^{\pi} \mathrm{d} \phi \cos (\phi) \int_{0}^{\pi} \mathrm{d} \theta \cos (\theta) R\left(\phi, \theta ; \boldsymbol{\mu}_{k}\right) \\
& M_{c s}\left(\boldsymbol{\mu}_{k}, \mathbf{U}_{k}\right)=\int_{-\pi}^{\pi} \mathrm{d} \phi \cos (\phi) \int_{0}^{\pi} \mathrm{d} \theta \sin (\theta) R\left(\phi, \theta ; \boldsymbol{\mu}_{k}, \mathbf{U}_{k}\right) \\
& M_{s c}\left(\boldsymbol{\mu}_{k}, \mathbf{U}_{k}\right)=\int_{-\pi}^{\pi} \mathrm{d} \phi \sin (\phi) \int_{0}^{\pi} \mathrm{d} \theta \cos (\theta) R\left(\phi, \theta ; \boldsymbol{\mu}_{k}, \mathbf{U}_{k}\right) \\
& M_{s s}\left(\boldsymbol{\mu}_{k}, \mathbf{U}_{k}\right)=\int_{-\pi}^{\pi} \mathrm{d} \phi \sin (\phi) \int_{0}^{\pi} \mathrm{d} \theta \sin (\theta) R\left(\phi, \theta ; \boldsymbol{\mu}_{k}, \mathbf{U}_{k}\right) \\
& M\left(\boldsymbol{\mu}_{k}, \mathbf{U}_{k}\right)=\int_{-\pi}^{\pi} \mathrm{d} \phi \int_{0}^{\pi} \mathrm{d} \theta R\left(\phi, \theta ; \boldsymbol{\mu}_{\ell}, \mathbf{U}_{k}\right) \\
& R(\phi, \theta ; \boldsymbol{\mu}, \mathbf{U})=\log (2 \pi)+\log \left[\int_{0}^{\pi} \mathrm{d} \theta^{\prime}\left|\sin \left(\theta^{\prime}\right)\right| \exp \left(B^{c} \cos \left(\theta^{\prime}\right)+B^{s} \sin \left(\theta^{\prime}\right)\right)\right. \\
& \times \exp \left(\beta J \left(U_{13} \cos \left(\theta^{\prime}\right) \sin (\theta) \cos (\phi)+U_{23} \cos \left(\theta^{\prime}\right) \sin (\theta) \sin (\phi)\right.\right. \\
& \left.\left.\left.+U_{33} \cos \left(\theta^{\prime}\right) \cos (\theta)\right)\right) I_{0}\left(\sqrt{L_{a}^{2}\left(\phi, \theta, \theta^{\prime}, \boldsymbol{\mu}, \mathbf{U}\right)+L_{b}^{2}\left(\phi, \theta, \theta^{\prime}, \boldsymbol{\mu}, \mathbf{U}\right)}\right)\right] \text {. }
\end{aligned}
$$

Here $I_{0}(x)$ is the zeroth-order modified Bessel function and

$$
\begin{aligned}
& L_{a}\left(\phi, \theta, \theta^{\prime}, \boldsymbol{\mu}, \mathbf{U}\right)=A^{c}+H^{c c} \cos \left(\theta^{\prime}\right)+H^{c s} \sin \left(\theta^{\prime}\right) \\
& \quad+\beta J\left[U_{11} \sin (\theta) \cos (\phi) \sin \left(\theta^{\prime}\right)+U_{21} \sin (\theta) \sin (\phi) \sin \left(\theta^{\prime}\right)+U_{31} \cos (\theta) \sin \left(\theta^{\prime}\right)\right] \\
& \begin{aligned}
L_{b}\left(\phi, \theta, \theta^{\prime}, \boldsymbol{\mu},\right. & \mathbf{U})=A^{s}+H^{s c} \cos \left(\theta^{\prime}\right)+H^{s s} \sin \left(\theta^{\prime}\right) \\
& +\beta J\left[U_{12} \sin (\theta) \cos (\phi) \sin \left(\theta^{\prime}\right)+U_{22} \sin (\theta) \sin (\phi) \sin \left(\theta^{\prime}\right)+U_{32} \cos (\theta) \sin \left(\theta^{\prime}\right)\right] .
\end{aligned}
\end{aligned}
$$

\section{References}

[1] Viana L and Bray A J 1985 J. Phys. C: Solid State Phys. 183037

[2] Kanter I and Sompolinsky H 1987 Phys. Rev. Lett. 58164

[3] Mezard M and Parisi G 1987 Europhys. Lett. 31067

[4] Mottishaw P and De Dominicis C 1987 J. Phys. A: Math. Gen. 20 L375

[5] Wong K Y and Sherrington D 1988 J. Phys. A: Math. Gen. 21 L459

[6] Monasson R 1998 J. Phys. A: Math. Gen. 31513

[7] Mezard M and Parisi G 2001 Eur. Phys. J. B 20217

[8] Mezard M and Parisi G 2003 J. Stat. Phys. 1111

[9] Wemmenhove B, Nikoletopoulos T and Hatchett J P L 2004 Preprint cond-mat/0405563

[10] Murayama T, Kabashima Y, Saad D and Vicente R 2000 Phys. Rev. E 621577

[11] Nakamura K, Kabashima Y and Saad D 2001 Europhys. Lett. 56610

[12] Nishimori H 2001 Statistical Physics of Spin Glasses and Information Processing (Oxford: Oxford University Press)

[13] Skantzos N S, van Mourik J, Saad D and Kabashima Y 2003 J. Phys. A: Math. Gen. 3611131

[14] Kirkpatrick S and Selman B 1994 Science 2641297

[15] Monasson R and Zecchina R 1998 Phys. Rev. E 561357

[16] Monasson R and Zecchina R 1998 J. Phys. A: Math. Gen. 319209

[17] Monasson R, Zecchina R, Kirkpatrick S, Selman B and Troyansky L 1999 Nature 400133 
[18] Wemmenhove B and Coolen A C C 2003 J. Phys. A: Math. Gen. 369617

[19] Pérez Castillo I and Skantzos N S 2004 J. Phys. A: Math. Gen. 379087

[20] Pérez Castillo I, Wemmenhove B, Hatchett J P L, Coolen A C C, Skantzos N S and Nikoletopoulos T 2004 J. Phys. A: Math. Gen. 378789

[21] Nikoletopoulos T, Coolen A C C, Pérez Castillo I, Skantzos N S, Hatchett J P L and Wemmenhove B 2004 J. Phys. A: Math. Gen. 376455

[22] Semerjian G and Cugliandolo L F 2003 Europhys. Lett. 61247

[23] Semerjian G and Weigt M 2004 J. Phys. A: Math. Gen. 375525

[24] Hatchett J P L, Wemmenhove B, Pérez Castillo I, Nikoletopoulos T, Skantzos N S and Coolen A C C 2004 J. Phys. A: Math. Gen. 376201

[25] Hatchett J P L, Pérez Castillo I, Coolen A C C and Skantzos N S 2005 Preprint cond-mat/0504313

[26] Sherrington D and Kirkpatrick S 1975 Phys. Rev. Lett. 351792

[27] Schuster H G and Wagner P 1990 Biol. Cybern. 6477

[28] Schuster H G and Wagner P 1990 Biol. Cybern. 6483

[29] Abbott L F 1990 J. Phys. A: Math. Gen. 233835

[30] Acebrón J A, Bonilla L L, Pérez-Vicente C J, Ritort F and Spigler R 2005 Rev. Mod. Phys. 77 137-85

[31] Choi M Y and Doniach S 1985 Phys. Rev. B 314516

[32] Wiesenfeld K, Colet P and Strogatz S H 1996 Phys. Rev. Lett. 76404

[33] Wiesenfeld K, Colet P and Strogatz S H 1998 Phys. Rev. E 571563

[34] Granato E, Kosterlitz J M and Nightingale M P 1996 Physica B 222266

[35] Majhofer A 1996 Physica B 222273

[36] Houghton A and Moore M A 1988 Phys. Rev. B 385045

[37] Marinari E, Parisi G and Ritort F 1995 J. Phys. A: Math. Gen. 284481

[38] Tang L-H 1996 Phys. Rev. B 543350

[39] Coolen A C C and Pérez-Vicente C J 2003 J. Phys. A: Math. Gen. 364477

[40] Parisi G 1979 Phys. Lett. A 73203

[41] Parisi G and Toulouse G 1980 J. Phys. Lett. (Paris) 41 L361

[42] Abramowitz M and Stegun I A (ed) 1972 Handbook of Mathematical Functions (New York: Dover)

[43] Jalan S and Amritkar R E 2004 Physica A 321220

[44] Miyatake Y, Yamamoto M, Kim J J, Toyonaga M and Nagai O 1986 J. Phys. C: Solid State Phys. 192539

[45] Loison D, Qin C L, Schotte K D and Jin X F 2004 Eur. Phys. J. B 41395 